\title{
SPECIAL HAGEMANN RELATIONS AND CONGRUENCE $n$-PERMUTABLE VARIETIES
}

\author{
ALBERTO CHICCO \\ Department of Mathematics and Statistics \\ McMaster University \\ Hamilton, ON \\ Canada \\ e-mail: chiccoa@mcmaster.ca
}

\begin{abstract}
The aim of this paper is to present some new results concerning the property of congruence $n$-permutability for varieties of algebras. The first of such results is a new termwise characterization of congruence $n$-permutability, obtained by using some relations with particular configurations. Indeed, the shapes of these relations have inspired the definition of what we have called special Hagemann relations of dimension $k$, briefly $S H R_{k}$ : for fixed $k \geq 1$, the class of varieties that omit $S H R_{k}$ turns out to be a prime Maltsev filter within the lattice of interpretability types (see [5]). Finally, by still using the special Hagemann relations, we provide some arguments for the primeness of:

(1) congruence 2-permutability with respect to idempotent varieties;

(2) congruence 3-permutability with respect to idempotent locally finite varieties (more thoroughly discussed in [3]);

(3) congruence $n$-permutability, for some $n \geq 2$, with respect to idempotent varieties.
\end{abstract}

2010 Mathematics Subject Classification: 08B05, 03C05.

Keywords and phrases: congruence $n$-permutable variety, idempotent, Maltsev condition, prime, special Hagemann relation.

Received June 21, 2019

(C) 2019 Scientific Advances Publishers 


\section{Introduction}

The property of being congruence $n$-permutable $(n \geq 2)$ for a variety of algebras $\mathcal{V}$ is a particular feature of the congruence lattices of the algebras in $\mathcal{V}$ that has been thoroughly studied throughout the past decades in the field of Universal Algebra, along with the notion of (strong) Maltsev condition. As a matter of fact, these two central concepts have been historically related ever since Maltsev provided in [12] the famous characterization of congruence 2-permutable varieties (also referred to simply as congruence permutable varieties) by the existence of a certain term (called Maltsev term) satisfying a pair of equations; for this reason, congruence permutability is considered as a sort of template of what a Maltsev condition represents. More precisely, his argument was based on the fact that, given a variety $\mathcal{V}$, the existence of a term $m$ in the language of $\mathcal{V}$, satisfying $m(x, y, y) \approx m(y, y, x) \approx x$, forces the congruences of any algebra $\mathbf{A}$ in $\mathcal{V}$ to permute, i.e., to satisfy $\theta \circ \varphi=\varphi \circ \theta$, for all $\theta, \varphi \in \operatorname{Con}(\mathbf{A})$. Indeed, if $(a, b) \in \theta \circ \varphi$, with $c \in A$ being an element so that $(a, c) \in \theta$ and $(c, b) \in \varphi$, then the term operation $m^{\mathbf{A}}$ completes the inverse connection as follows:

$$
a=m^{\mathbf{A}}(a, c, c) \varphi m^{\mathbf{A}}(a, c, b) \theta m^{\mathbf{A}}(c, c, b)=b .
$$

This implies $(a, b) \in \varphi \circ \theta$. Surprisingly, the converse also holds, that is the existence of a Maltsev term is forced by the property of congruence permutability of the variety $\mathcal{V}$. In detail, since the free algebra $\mathbf{F}$ of $\mathcal{V}$ generated by $\{x, y, z\}$ is congruence permutable and because $(x, z) \in \mathrm{Cg}^{\mathbf{F}}(x, y) \circ \mathrm{Cg}^{\mathbf{F}}(y, z)$, then there must exist an element of the free algebra, namely a ternary term $m$ in the language of $\mathcal{V}$, satisfying $\quad(x, m(x, y, z)) \in \mathrm{Cg}^{\mathbf{F}}(y, z) \quad$ and $\quad(m(x, y, z), z) \in \mathrm{Cg}^{\mathbf{F}}(x, y)$. These relationships yield that the term $m$ has to satisfy $m(x, y, y) \approx x$ and $m(x, x, z) \approx z$ throughout the entire variety, implying the existence of a Maltsev term for $\mathcal{V}$. This way of reasoning has become rather standard in the study of Maltsev conditions and has led to the discovery of many other interesting characterizations of certain properties of varieties. 
After the previous brief presentation and before we proceed further, we need to provide some basic definitions for which we mostly refer to [13] and [5]. By an algebra $\mathbf{A}$ we mean a pair $\langle A ; F\rangle$, where $\mathbf{A}$ is a nonempty set, called the universe of $\mathbf{A}$, and $F=\left\{f_{i}: i \in I, f_{i}: A^{n_{i}} \rightarrow A\right.$, $\left.n_{i} \geq 0\right\}$ is a set of operations on $A$, called the basic operations of $\mathbf{A}$. Moreover, a term operation of $\mathbf{A}$ is an operation on $A$ obtained by composition of basic operations of $\mathbf{A}$ and, possibly, the projections; a term operation $t^{\mathbf{A}}$ of arity $k$ is idempotent if $t^{\mathbf{A}}\left(\{a\}^{k}\right)=\{a\}$, for all $a \in A$, and we will say that an algebra (resp., a class of algebras) is idempotent, if all of its basic operations (resp., all of its members) are. Associated to every algebra A, we can always build the set of equivalence relations on A which are compatible with the basic operations of $\mathbf{A}$, called the congruences of $\mathbf{A}$. Such a set is referred to as $\operatorname{Con}(\mathbf{A})$ and can be endowed with a binary operation $\Lambda=\bigcap$ (the standard intersection), called meet, and another binary operation $\vee$, called join, defined as the transitive closure of the union of two congruences; the so obtained structure is a lattice, called the congruence lattice of $\mathbf{A}$ and denoted by $\operatorname{Con}(\mathbf{A})$. An alternative way to describe the join of two congruences of an algebra is through the following notions.

Given a set $A$ and $R, S \subseteq A \times A$, the composition or relational product of $R$ and $S$ is the binary relation $R \circ S \subseteq A \times A$ defined by

$$
R \circ S=\{(a, c): \exists b \in A[(a, b) \in R,(b, c) \in S]\} .
$$

We can also inductively define, for $n \geq 1$, the iterated composition as shown below:

$$
\begin{gathered}
R \circ^{1} S=R ; \\
R \circ^{n+1} S=R \circ\left(S \circ^{n} R\right) .
\end{gathered}
$$

Furthermore, we will make use of the notation $a / E:=\{b:(a, b) \in E\}$ and $E^{-1}:=\{(y, x):(x, y) \in E\}$, for $E \subseteq A \times B$ and $a \in A$. 
These being defined, it is a known fact that, for an algebra $\mathbf{A}$ and for all $\alpha, \beta \in \operatorname{Con}(\mathbf{A})$,

$$
\alpha \vee \beta=\bigcup_{n \geq 1} \alpha \circ^{n} \beta
$$

However, there exist some algebras where the previous union has finitely many terms or, in other words, where there is some $n \geq 2$ such that $\alpha \vee \beta=\alpha \circ^{n} \beta$, for all congruences $\alpha, \beta$ : if this is the case, we say that $\alpha$ and $\beta$ n-permute and that the algebra is congruence $n$-permutable. Equivalently, any two congruences $\alpha, \beta$ of a congruence $n$-permutable algebra A satisfy $\alpha{ }^{n} \beta=\beta{ }^{n} \alpha$. Also, we say that a class of algebras is congruence n-permutable if all of its members are.

As far as classes of algebras are concerned, a variety is a class of similar algebras which contains all the homomorphic images, subalgebras and direct products of its members. Sometimes, a variety is referred to as an equational class and this is due to Birkhoff's HSP theorem, which states that a class of similar algebras is a variety if and only if it is the class of models of some set of equations (an equation is a universally quantified sentence of the form $\forall \bar{x}[p(\bar{x}) \approx q(\bar{x})]$, usually denoted simply by $p(\bar{x}) \approx q(\bar{x})$, where $p$ and $q$ are terms in some fixed language). We can rephrase this by saying that any variety $\mathcal{V}$ is of the form $\mathcal{V}=\operatorname{Mod}\left(\sum\right)$, for some suitable set of equations $\sum$ also referred to as an axiomatization of $\mathcal{V}$. It is worth mentioning, for the sake of this paper, that varieties have been naturally classified via the notion of interpretability (for a reference, see [5]): given any two varieties $\mathcal{V}$ (having set of basic operation symbols $F$ ) and $\mathcal{W}$, we say that $\mathcal{V}$ is interpretable in $\mathcal{W}$, and write $\mathcal{V} \preceq \mathcal{W}$, if for every basic operation symbol $h \in F$ of $\mathcal{V}$, there is a corresponding term $\iota(h)$ of $\mathcal{W}$, such that, for each algebra $\mathbf{A} \in \mathcal{W}$, the algebra $\left\langle A ;\left\{\iota(h)^{\mathbf{A}}\right\}_{h \in F}\right\rangle$ lies in $\mathcal{V}$, and the arity of 
( $(h)$ equals the arity of $h$ whenever this is at least 1 ; otherwise, if $h$ has arity 0 , then $\iota(h)$ is a unary term satisfying the equation $\iota(h)(x) \approx$ $\iota(h)(y)$. Whenever two varieties $\mathcal{V}$ and $\mathcal{W}$ satisfy $\mathcal{V} \preceq \mathcal{W} \preceq \mathcal{V}$, then we say that they are equi-interpretable. As an equivalence relation, the relation of equi-interpretability partitions the class of all varieties into equivalence classes which have been given the name of interpretability types. The class $L$ of interpretability types can also be partially ordered by defining that the interpretability type of a variety $\mathcal{V}$ is " $\leq$ " the interpretability type of $\mathcal{W}$ if and only if $\mathcal{V} \preceq \mathcal{W}$ : with such an order, the class $L$ becomes a poset which is, in fact, a lattice $\mathbf{L}$, called the lattice of interpretability types. If we consider the class of interpretability types containing some idempotent variety, we obtain a sublattice of $\mathbf{L}$ that we denote by $\mathbf{L}^{i d}$. For convenience, from this point on, we will no longer distinguish any variety from its interpretability type and we will always use the notation $\mathcal{V} \leq \mathcal{W}$, rather than $\mathcal{V} \preceq \mathcal{W}$ (in other words we deal with varieties up to equi-interpretability). At this point, it is worth providing a description of the join and the meet of two varieties, which will be needed eventually, and we provide them as presented in [19] and [18]. For any pair of varieties $\mathcal{V}$ and $\mathcal{W}$ having distinct sets of operation symbols $I$ and $J$ respectively, the following holds:

$\mathcal{V} \vee \mathcal{W}=\left\{\left\langle A ;\left\{f_{i}: i \in I \cup J\right\}\right\rangle:\left\langle A ;\left\{f_{i}: i \in I\right\}\right\rangle \in \mathcal{V},\left\langle A ;\left\{f_{i}: i \in J\right\}\right\rangle \in \mathcal{W}\right\}$

$$
\mathcal{V} \wedge \mathcal{W}=\{\mathbf{A} \otimes \mathbf{B}: \mathbf{A} \in \mathcal{V}, \mathbf{B} \in \mathcal{W}\}
$$

where $\mathbf{A} \otimes \mathbf{B}$ denotes the non-indexed product of $\mathbf{A}$ and $\mathbf{B}$, namely, the algebra with universe $A \times B$ and basic operations given by all possible operations of the form $f^{\mathbf{A}} \times g^{\mathbf{B}}:(A \times B)^{k} \rightarrow A \times B$, where $\left(f^{\mathbf{A}} \times g^{\mathbf{B}}\right)$ $\left(\left(a_{1}, b_{1}\right), \ldots,\left(a_{k}, b_{k}\right)\right)=\left(f^{\mathbf{A}}\left(a_{1}, \ldots, a_{k}\right), g^{\mathbf{B}}\left(b_{1}, \ldots, b_{k}\right)\right), a_{i} \in A, b_{i} \in B$, for all $i=1, \ldots, k$, and $f^{\mathbf{A}}$ and $g^{\mathbf{B}}$ are $k$-ary term operations of, respectively, $\mathbf{A}$ and $\mathbf{B}$. 
A variety $\mathcal{V}$ is finitely presentable if it has finitely many basic operation symbols and there exists a finite axiomatization for it. The interpretability type of a finitely presentable variety is called a strong Maltsev condition and a countable family of strong Maltsev conditions $\left\{\mathcal{V}_{n}: n<\omega\right\}$ with $\mathcal{V}_{i+1} \leq \mathcal{V}_{i}$, for all $i<\omega$, is called a Maltsev condition; moreover, the filter they give rise to is referred to as a (strong) Maltsev class. We say that a (strong) Maltsev class $\mathfrak{E}$ is prime if, whenever $\mathcal{V} \vee \mathcal{W} \in \mathfrak{E}$, for every $\mathcal{V}, \mathcal{W} \in L$, then either $\mathcal{V} \in \mathfrak{E}$ or $\mathcal{W} \in \mathfrak{E}$. Likewise, we say that $\mathfrak{E}$ is prime with respect to idempotent varieties if the above property holds for the subclass $\mathfrak{E} \cap L^{i d}$ and the varieties $\mathcal{V}$ and $\mathcal{W}$ belong to $L^{i d}$.

We close this section with the statement of a criterion, proven by Taylor in [18], that characterizes Maltsev classes and that we are going to make use of later on.

Theorem 1.1 ([18]). Let $\mathfrak{E}$ be a non-empty class of (interpretability types of ) varieties. Then, $\mathfrak{E}$ is a [strong] Maltsev class if and only if the following independent statements hold for $\mathfrak{E}$, where $\mathcal{V}, \mathcal{W}, \mathcal{V}_{i}$ are any varieties, $i<\kappa$ and $\kappa>0$ is a cardinal:

(1) if $\mathcal{W}$ and $\mathcal{V}$ are equi-interpretable and $\mathcal{V} \in \mathfrak{E}$, then $\mathcal{W} \in \mathfrak{E}$;

(2) if $\mathcal{W}$ is a subvariety of $\mathcal{V}$ and $\mathcal{V} \in \mathfrak{E}$, then $\mathcal{W} \in \mathfrak{E}$;

(3) if $\left\{\mathcal{V}_{i}: i<\kappa\right\} \subseteq \mathfrak{E}$ and $\kappa$ is finite [arbitrarily large], then $\bigwedge_{i<\kappa} \mathcal{V}_{i}$ $\in \mathfrak{E}$

(4) if $\operatorname{Mod}\left(\sum\right) \in \mathfrak{E}$, then there exists a finite subset $\Delta \subseteq \sum$ such that $\operatorname{Mod}(\Delta)$ is similar to $\operatorname{Mod}\left(\sum\right)$ and $\operatorname{Mod}(\Delta) \in \mathfrak{E}$.

For further and deeper explanations of the last concepts so far discussed, refer to [5] and [14]. 
By using most of the aforementioned concepts and tools, we are going to show that the subclass of $L$ containing all and only those (interpretability types of) varieties in which no special Hagemann relations of a fixed dimension $n \geq 1$ occur (which will be defined in one of the next sections), denoted by $\Omega\left(S H R_{n}\right)$, is a prime Maltsev class which in turn contains all congruence $(n+1)$-permutable varieties. A natural question that may be asked is whether $\Omega\left(S H R_{n}\right)$ coincides with the class $C P_{n+1}$ of congruence $(n+1)$-permutable varieties: in fact, we are able to answer this question negatively, although we can still argue that $\Omega\left(S H R_{k}\right) \cap L^{i d}=C P_{1+k} \cap L^{i d}$, for $k \in\{1, \omega\}$. This latter fact will also represent a primeness argument for congruence 2-permutability with respect to idempotent varieties, as well as for congruence $n$-permutability, for some $n \geq 2$, with respect to idempotent varieties.

\section{A New Maltsev Characterization for Congruence $n$-Permutability}

We mentioned at the very beginning of this paper that the first termwise characterization of congruence permutability was exhibited in 1954 by Maltsev in [12], where he essentially proved that a variety $\mathcal{V}$ is congruence permutable if and only if $\operatorname{Mod}(\{m(x, y, y) \approx x, m(x, x, y) \approx$ $y\}) \leq \mathcal{V}$. Some generalizations of Maltsev's argument to congruence $n$-permutability for all values of $n \geq 2$ appeared a few years later and were developed under different perspectives. The first of these was given in 1969 by Schmidt in [16], where he simulated Maltsev's already existing approach, being able to generalize it by using a pair of suitable congruences of the $(n+1)$-generated free algebra of an $n$-permutable variety, so as to finally produce the so called Schmidt terms, satisfying certain equations. On the other hand, Hagemann and Mitschke provided in 1973 a more useful characterization of congruence $n$-permutability involving $n-1$ ternary terms, ever since called Hagemann-Mitschke terms ([6]). Their argument was based on considering some suitable 
reflexive compatible relations satisfying a certain condition. In the next theorem, we are going to list all the crucial aspects of the aforementioned results, so that to provide a synthesis of the achievements obtained during the first decades of analysis of congruence $n$-permutable varieties.

Theorem 2.1. Let $n>1, \mathcal{V}$ be any variety and let $\mathbf{F}_{k}$ denote the free algebra of $\mathcal{V}$ generated by $\left\{x_{1}, \ldots, x_{k}\right\}$, for $k \geq 1$. Then the following are equivalent:

(1) $\mathcal{V}$ is congruence n-permutable;

(2) [16] $\mathbf{F}_{n+1}$ is congruence n-permutable;

(3) [6] For all $\mathbf{A} \in \mathcal{V}$ and $R \leq \mathbf{A}^{2}$, if $R$ is reflexive, then $R^{-1} \subseteq R{ }^{n-1}$ $R$;

(4) [6] If $S=\operatorname{Sg}^{F_{2}^{2}}\left(\left\{\left(x_{1}, x_{1}\right),\left(x_{1}, x_{2}\right),\left(x_{2}, x_{2}\right)\right\}\right)$, then $\left(x_{2}, x_{1}\right) \in S$ ${ }^{n-1} S$;

(5) [16] (Schmidt terms) $\mathcal{S}_{n} \leq \mathcal{V}$, where $\mathcal{S}_{n}$ is the variety with $(n+1)$-ary basic operation symbols $\left\langle s_{0}, \ldots, s_{n}\right\rangle$, axiomatized by:

$$
\begin{aligned}
x_{0} & \approx s_{0}\left(x_{0}, \ldots, x_{n}\right) \\
s_{i}\left(x_{0}, x_{0}, x_{2}, x_{2}, \ldots\right) & \approx s_{i+1}\left(x_{0}, x_{0}, x_{2}, x_{2}, \ldots\right) \text { for odd } i \\
s_{i}\left(x_{0}, x_{1}, x_{1}, x_{3}, x_{3}, \ldots\right) & \approx s_{i+1}\left(x_{0}, x_{1}, x_{1}, x_{3}, x_{3}, \ldots\right) \text { for even } i \\
s_{n}\left(x_{0}, \ldots, x_{n}\right) & \approx x_{n} ;
\end{aligned}
$$

(6) [6] (Hagemann-Mitschke terms) $\mathcal{H M}_{n} \leq \mathcal{V}$, where $\mathcal{H M}_{n}$ is the variety with ternary basic operation symbols $\left\langle p_{1}, \ldots, p_{n-1}\right\rangle$, axiomatized by:

$$
\begin{aligned}
x & \approx p_{1}(x, y, y) \\
p_{i}(x, x, y) & \approx p_{i+1}(x, y, y) \text { for } 1 \leq i \leq n-1 \\
p_{n-1}(x, x, y) & \approx y .
\end{aligned}
$$


A relation $R$ that does not satisfy the statement (3) of Theorem 2.1 has been called in [4] an $(n-1)$-dimensional Hagemann relation. More precisely:

Definition 2.1. Given $k \geq 1$ and an algebra A, a Hagemann relation of dimension $k$ or, equivalently, a $k$-dimensional Hagemann relation, is a binary reflexive subuniverse $R \leq \mathrm{A}^{2}$, such that $R^{-1} \nsubseteq R \circ^{k} R$.

The equivalence of (1) and (3) in Theorem 2.1 can be rephrased by saying that, for any $n \geq 2$, a variety is congruence $n$-permutable if and only if it contains no algebras carrying any Hagemann relations of dimension $n-1$. In particular, Hagemann and Mitschke implicitly used statement (4) of Theorem 2.1 to find the strong Maltsev condition displayed in statement (6) of the same theorem (we are going to show an alternative semantic proof of the equivalence of (1) and (3) in Section 3).

Next, we present a theorem that displays a new strong Maltsev condition for congruence $n$-permutability of varieties. We provide further comments after the proof.

Theorem 2.2. Let $n>1$ and $\mathcal{V}$ be a variety. The following statements are equivalent:

(1) $\mathcal{V}$ is congruence n-permutable;

(2) $\mathcal{X}_{n} \leq \mathcal{V}$, where $\mathcal{X}_{n}$ is the variety with $k_{n}$-ary basic operation symbols $\left\langle t_{1}, \ldots, t_{n-1}\right\rangle$, with $k_{n}=\frac{n^{2}+5 n+2}{2}$, axiomatized by:

$$
\begin{aligned}
x_{0} & \approx t_{1}(\vec{v}) ; \\
t_{i}(\vec{w}) & \approx t_{i+1}(\vec{v}) \text { for } 1 \leq i<n-1 ; \\
t_{n-1}(\vec{w}) & \approx x_{n} ;
\end{aligned}
$$


where $\vec{v}, \vec{w} \in\left\{x_{0}, \ldots, x_{n}\right\}^{k_{n}}$ are defined as

$$
\begin{aligned}
& \vec{v}=(\underbrace{x_{0}, \ldots, x_{n}}_{n+1}, \underbrace{x_{0}, \ldots, x_{n}}_{n+1}, \underbrace{x_{1}, \ldots, x_{n}}_{n}, \underbrace{x_{2}, \ldots, x_{n}}_{n-1}, \ldots, x_{n-1}, x_{n}) ; \\
& \vec{w}=(\underbrace{x_{0}, \ldots, x_{0}}_{n+1}, \underbrace{x_{1}, \ldots, x_{1}}_{n+1}, \underbrace{x_{2}, \ldots, x_{2}}_{n}, \underbrace{x_{3}, \ldots, x_{3}}_{n-1}, \ldots, x_{n}, x_{n}) .
\end{aligned}
$$

Proof. Fix any integer $n>1$.

$(1) \Rightarrow(2)$ : Let $\mathcal{V}$ be a congruence $n$-permutable variety and call $\mathbf{F}$ the free algebra in $\mathcal{V}$ generated by $X:=\left\{x_{0}, \ldots, x_{n}\right\}$. Define the following set of pairs of variables:

$$
V=\left(\left\{x_{0}, x_{1}\right\} \times X\right) \cup \bigcup_{i=2}^{n}\left(\left\{x_{i}\right\} \times\left\{x_{i-1}, \ldots, x_{n}\right\}\right) .
$$

$V$ is a binary relation on the set $X$, where $x_{0} / V=x_{1} / V=X$ and, for $2 \leq i \leq n, x_{i} / V=X-\left\{x_{0}, \ldots, x_{i-2}\right\}$, therefore, an easy computation yields that

$$
|V|=\frac{n^{2}+5 n+2}{2}
$$

Call $k_{n}:=|V|$. Consider now the subuniverse $R$ of $\mathbf{F}^{2}$ defined by

$$
R=S g^{\mathbf{F}^{2}}(V)
$$

Since $V$ contains $\left(x_{i}, x_{i}\right)$ for all $0 \leq i \leq n, R$ is reflexive. Hence, by Theorem 2.1(3), we have that $R^{-1} \subseteq R \circ^{n-1} R$ and, because $\left(x_{0}, x_{n}\right) \in R$ by definition of $V$, we deduce that there must exist $u_{1}, \ldots$, $u_{n-2} \in F$ such that

$$
x_{n}=: u_{n-1} R u_{n-2} R \cdots R u_{1} R u_{0}:=x_{0} .
$$


Let $t_{i}^{\mathbf{F}}(1 \leq i \leq n-1)$ be the $k_{n}$-ary term operation of $\mathbf{F}$ such that $\left(u_{i}, u_{i-1}\right) \in t_{i}^{\mathbf{F}}(V)$. Also, if $\vec{v}$ and $\vec{w}$ are defined as in the statement (2), then we have that

$$
V=\bigcup_{i=1}^{k_{n}}\left\{\left(\pi_{i}(\vec{w}), \pi_{i}(\vec{v})\right)\right\} .
$$

Therefore, if we order the pairs in $V$ according to $\vec{v}$ and $\vec{w}$, we obtain

$$
\begin{aligned}
x_{n} & =t_{n-1}^{\mathbf{F}}(\vec{w}) ; \\
t_{i}^{\mathbf{F}}(\vec{v}) & =u_{i}=t_{i-1}^{\mathbf{F}}(\vec{w}) \text { for } 2 \leq i \leq n-1 ; \\
t_{1}^{\mathbf{F}}(\vec{v}) & =x_{0} .
\end{aligned}
$$

These identities being satisfied in $\mathbf{F}$ yields that the entire variety $\mathcal{V}$ satisfies them, proving that $\mathcal{X}_{n} \leq \mathcal{V}$.

(2) $\Rightarrow$ (1): Assume $\mathcal{V}$ has terms $t_{1}, \ldots, t_{n-1}$ satisfying (2). Let $\mathbf{A} \in \mathcal{V}$ and $R \leq \mathbf{A} \times \mathbf{A}$ be a reflexive subuniverse. For any $(a, b) \in R$, we are going to define some elements of $A$ using the terms $t_{1}, \ldots, t_{n-1}$ in the following way:

For $1 \leq i \leq n-1$, let

$$
\begin{aligned}
& d_{i}=t_{i}^{\mathbf{A}}(\overbrace{a, \ldots, a}^{n+1}, \overbrace{a, \ldots, a}^{n+1}, \overbrace{a, \ldots, a}^{n}, \ldots, \overbrace{a, \ldots, a}^{n-i+2}, \overbrace{b, \ldots, b}^{n-i+1}, \ldots, \overbrace{b, b}^{2}) ; \\
& e_{i}=t_{i}^{\mathbf{A}}(\overbrace{\underbrace{a, \ldots, a}_{i}, b, \ldots, b}^{n+1}, \overbrace{\underbrace{a, \ldots, a}_{i}, b, \ldots, b}^{\overbrace{i}}, \overbrace{\underbrace{a, \ldots, a}_{i-1}, b, \ldots, b}^{\overbrace{i}}, \\
& \ldots \underbrace{\overbrace{a, b}, \ldots, b}_{1}, \overbrace{b, \ldots, b}^{n-i+2}, \ldots, \overbrace{b, b}^{2}) .
\end{aligned}
$$


Since $(a, a),(b, b) \in R$ ( $R$ is reflexive) and $(a, b) \in R$, we observe that $\left(d_{i}, e_{i}\right) \in R$, for $1 \leq i \leq n-1$. Moreover, notice that the equations satisfied by the $t_{i}$ 's yield the following:

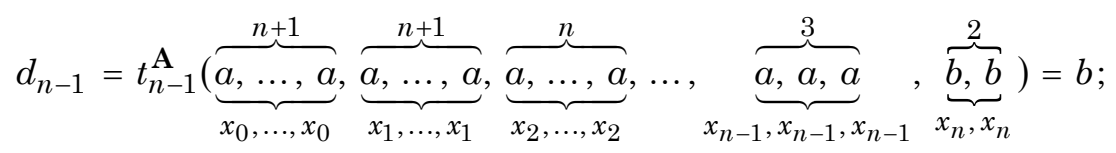

for $2 \leq i \leq n-1$,

$$
\begin{aligned}
& e_{i}=t_{i}^{\mathbf{A}}(\overbrace{\underbrace{}_{x_{0}, \ldots, x_{i-1}, x_{i}, \ldots, x_{n}}}^{n+1}, \underbrace{n+1}_{x_{0}, \ldots, x_{i-1}, x_{i}, \ldots, x_{n}}, \underbrace{\overbrace{a, \ldots, a, b, a, b, \ldots, b}^{n+1}}_{x_{1}, \ldots, x_{i-1}, x_{i}, \ldots, x_{n}},
\end{aligned}
$$

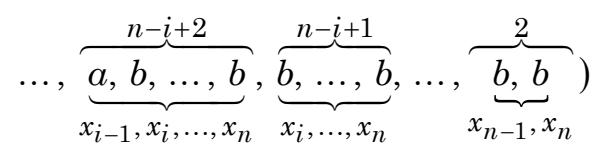

$$
\begin{aligned}
& =t_{i-1}^{\mathbf{A}}(\overbrace{\underbrace{}_{x_{0}, \ldots, x_{0}} \overbrace{a, \ldots, a}^{n+1}}^{\overbrace{\underbrace{}_{x_{1}, \ldots, x_{1}}, \ldots, a}^{n+1}}, \overbrace{\underbrace{\overbrace{a, \ldots, a}}_{x_{2}, \ldots, x_{2}}}^{n}, \ldots, \overbrace{\underbrace{a, \ldots, a}_{x_{i-1}, \ldots, x_{i-1}}}^{n-i+3}, \overbrace{\underbrace{n, \ldots, b}_{x_{i}, \ldots, x_{i}}}^{n-i+2}, \ldots, \overbrace{\underbrace{b, b}_{x_{n}, x_{n}}}^{2})=d_{i-1} ;
\end{aligned}
$$

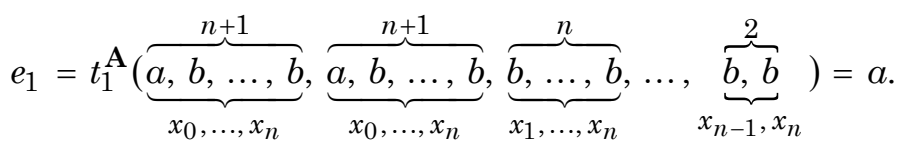

Therefore,

$$
b=d_{n-1} R e_{n-1}=d_{n-2} R \cdots R e_{2}=d_{1} R e_{1}=a,
$$

with $n-1$ occurrences of $R$, proving that $(b, a) \in R \circ^{n-1} R$.

By the arbitrariness of $R$ and $(a, b) \in R$, Theorem 2.1 guarantees that $\mathcal{V}$ is congruence $n$-permutable, completing the proof.

For any variety $\mathcal{V}$, if $\mathcal{X}_{n} \leq \mathcal{V}(n \geq 2)$, the terms that interpret $t_{1}, \ldots$, $t_{n-1}$ are called $\mathcal{X}_{n}$-terms. It is implied by Theorem 2.1 that, for fixed $n \geq 2$, the varieties $\mathcal{S}_{n}, \mathcal{H M}_{n}$ and $\mathcal{X}_{n}$ are equi-interpretable to each other. More precisely, the respective interpretations, for any fixed $n \geq 2$, are listed below. 
- $\mathcal{H} \mathcal{M}_{n} \leq \mathcal{S}_{n}$ (see also [6]): define the terms $p_{1}, \ldots, p_{n-1}$ by using $s_{1}, \ldots, s_{n-1}$, for $1 \leq i \leq n-1$ as follows:

$$
p_{i}(x, y, z):=s_{i}(\overbrace{x, \ldots, x}^{i}, y, \underbrace{z, \ldots, z}_{n-i}) .
$$

- $\mathcal{S}_{n} \leq \mathcal{H} \mathcal{M}_{n}$ (see also [6]): given $p_{1}, \ldots, p_{n-1}$, define $s_{0}, \ldots, s_{n}$ as

$$
\begin{aligned}
s_{0}\left(x_{0}, \ldots, x_{n}\right) & :=x_{0} \\
s_{i}\left(x_{0}, \ldots, x_{n}\right) & :=p_{i}\left(x_{i-1}, x_{i}, x_{i+1}\right) \text { for } 1 \leq i \leq n-1 \\
s_{n}\left(x_{0}, \ldots, x_{n}\right) & :=x_{n} .
\end{aligned}
$$

- $\mathcal{H} \mathcal{M}_{n} \leq \mathcal{X}_{n}$ : given $t_{1}, \ldots, t_{n-1}$, define the terms $p_{1}, \ldots, p_{n-1}$, as shown below

$$
\begin{gathered}
p_{i}(x, y, z):=t_{i}(\overbrace{\underbrace{x, \ldots, x}_{i}, y, \ldots, y}^{n+1}, \overbrace{\underbrace{x, \ldots, x}_{i}, y, \ldots, y}^{n+1}, \overbrace{\underbrace{x, \ldots, x}_{i-1}, y, \ldots, y}^{n}, \\
\ldots, \overbrace{x, y, \ldots, y}^{n-i+2}, \overbrace{z, \ldots, z}^{e_{i, n}}),
\end{gathered}
$$

for $1 \leq i \leq n-1$, where $e_{i, n}=\frac{n^{2}-(2 i-3) n+i^{2}-3 i}{2}=\sum_{j=i-1}^{n-2} n-j$.

- $\mathcal{X}_{n} \leq \mathcal{H} \mathcal{M}_{n}$ : define $t_{1}, \ldots, t_{n-1}$ by using $p_{1}, \ldots, p_{n-1}$, for $1 \leq i \leq n-1$, as follows

$$
t_{i}\left(x_{0}, \ldots, x_{k_{n}-1}\right):=p_{i}\left(x_{0}, x_{n}, x_{k_{n}-1}\right)
$$

One may observe that the arity of the $\mathcal{X}_{n}$-terms grows according to a second degree polynomial in $n$, which makes such terms rather inconvenient. We can absolutely claim that, so far, the most manageable terms characterizing congruence $n$-permutable varieties have been the Hagemann-Mitschke ones, although there also exists a way to capture 
the property of congruence $n$-permutability via a single term (as proven in [11]). Nonetheless, the way we have achieved the new description with the $\mathcal{X}_{n}$-terms makes use of a certain generating graph in some suitable free algebra (as we highlight in the next corollary of Theorem 2.2), that has inspired the topic discussed in Section 4.

Corollary 2.2.1. For $n>1$ and $\mathcal{V}$ a variety, $\mathcal{V}$ is congruence n-permutable if and only if $R_{n}^{-1} \subseteq R_{n}{ }^{\circ}{ }^{n-1} R_{n}$, where $R_{n}$ is the subuniverse of $\mathbf{F}_{\mathcal{V}}^{2}\left(\left\{x_{0}, \ldots, x_{n}\right\}\right)$ generated by

$$
V_{n}=\left(\left\{x_{0}, x_{1}\right\} \times\left\{x_{0}, \ldots, x_{n}\right\}\right) \cup \bigcup_{i=2}^{n}\left(\left\{x_{i}\right\} \times\left\{x_{i-1}, \ldots, x_{n}\right\}\right) .
$$

Therefore, the particular shape of the graph $\left\langle\left\{x_{0}, \ldots, x_{n}\right\} ; V_{n}\right\rangle$ is able to characterize the class of congruence $n$-permutable varieties, for every $n \geq 2$. As already mentioned, this fact has led us to considering some particular families of Hagemann relations that will be discussed in Section 4 .

\section{Failure Algebras and Hagemann Relations}

In the current section, we provide an alternative semantic proof to the equivalence of the statements (1) and (3) of Theorem 2.1. As a matter of fact, the original argument, invoked by Hagemann and Mitschke, needs the appeal to the 2-generated free algebra and the relation $S$ defined in Theorem 2.1. In order to develop our approach, we introduce a couple of constructions, a refinement of one of which has been used to show the primeness of congruence 3-permutability in the locally finite idempotent case (see [3]) and will be also recalled later for a proof of the primeness of congruence 2-permutability with respect to idempotent varieties. Thus, let us first present a few definitions that are needed for this topic. 
Definition 3.1. Let $n \geq 2, A$ and $B$ be two sets and $E \subseteq A^{2}$, $F, D \subseteq B^{2}$ and $G \subseteq B^{n}$. Let us define the relations $E \otimes F \subseteq(A \times B)^{2}$ and $F \star G \subseteq B^{n+1}$ as:

$$
\begin{gathered}
E \otimes F=\left\{\left(\left[\begin{array}{l}
a_{1} \\
b_{1}
\end{array}\right],\left[\begin{array}{l}
a_{2} \\
b_{2}
\end{array}\right]\right):\left(a_{1}, a_{2}\right) \in E,\left(b_{1}, b_{2}\right) \in F\right\}, \\
F \star G=\left\{\left(b_{0}, b_{1}, \ldots, b_{n}\right):\left(b_{0}, b_{1}\right) \in F,\left(b_{1}, \ldots, b_{n}\right) \in G\right\} .
\end{gathered}
$$

Furthermore, we may define by induction a suitable iteration of the operations $\otimes$ and $\star$, for $k \geq 1$, as:

$$
\begin{gathered}
E \otimes^{1} F=E, \\
E \otimes^{k+1} F=E \otimes\left(F \otimes^{k} E\right) ;
\end{gathered}
$$

and

$$
\begin{gathered}
F \star^{0} D=A, \\
F \star^{1} D=F, \\
F \star^{k+1} D=F \star\left(D \star{ }^{k} F\right) .
\end{gathered}
$$

Notice that $E \otimes \otimes^{2} F=E \otimes F$ and $F \star^{2} D=F \star D$. Moreover, if $\mathbf{A}$ is an algebra and $R, S \leq \mathbf{A} \times \mathbf{A}$, then for all $n \geq 1, R \otimes^{n} S \leq \mathbf{A}^{n} \times \mathbf{A}^{n}$ and, for all $n \geq 0, R \star^{n} S \leq \mathbf{A}^{n+1}$.

The next theorem exhibits a primitive positive construction of an $(n-1)$-dimensional Hagemann relation carried by a member of the variety generated by a failure of congruence $n$-permutability (i.e., a non$n$-permutable algebra), for any $n \geq 2$. 
Theorem 3.1. Let $n \geq 1$, A be an algebra and $\alpha, \beta \in \operatorname{Con}(\mathbf{A})$.

If $\alpha$ and $\beta$ do not $(n+1)$-permute, with $\beta \circ^{n+1} \alpha \nsubseteq \alpha \circ^{n+1} \beta$, then the subalgebra $\mathbf{B}$ of $\mathbf{A}^{n}$, whose universe is

$$
B=\beta \star^{n-1} \alpha,
$$

carries the relation $R \leq \mathbf{B} \times \mathbf{B}$, defined by

$$
R=B^{2} \cap\left((\beta \circ \alpha) \otimes^{n}(\alpha \circ \beta)\right)
$$

which is a Hagemann relation of dimension $n$.

Proof. Let $n \geq 1, \mathbf{A}$ an algebra with $\alpha, \beta \in \operatorname{Con}(\mathbf{A}), B \leq \mathbf{A}^{n}$ and $R \leq \mathbf{B} \times \mathbf{B}$ as defined in the statement. Let us first prove that $R$ is reflexive.

For every $\vec{b}=\left(a_{1}, \ldots, a_{n}\right) \in B$, we have that $\left(a_{i}, a_{i}\right) \in \beta \circ \alpha$ and $\left(a_{i}, a_{i}\right) \in \alpha \circ \beta$, for all $1 \leq i \leq n$, proving that $(\vec{b}, \vec{b}) \in R$.

Henceforth, we need to prove that $R^{-1} \nsubseteq R{ }^{n} R$. Since $\beta{ }^{n+1} \alpha \nsubseteq$ $\alpha \circ^{n+1} \beta$, there exists a pair $\left(a_{0}, a_{n+1}\right) \in \beta \circ^{n+1} \alpha$, with $\left(a_{0}, a_{n+1}\right) \notin \alpha$ ${ }_{\circ}^{n+1} \beta$. Call $a_{1}, \ldots, a_{n} \in A$ the elements such that

$$
\begin{gathered}
\left(a_{i}, a_{i+1}\right) \in \beta \text { for even } 0 \leq i \leq n \\
\left(a_{i}, a_{i+1}\right) \in \alpha \text { for odd } 0 \leq i \leq n .
\end{gathered}
$$

By definition of $B$, we have that the $n$-tuples $\vec{b}_{0}:=\left(a_{0}, a_{1}, \ldots, a_{n-1}\right)$, $\vec{b}_{n}:=\left(a_{2}, a_{3}, \ldots, a_{n+1}\right)$ are elements of $B$. Moreover,

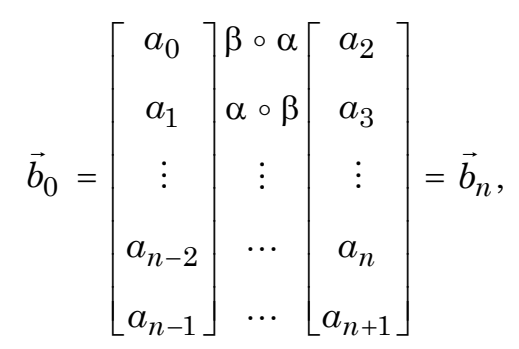

which actually means $\left(\vec{b}_{0}, \vec{b}_{n}\right) \in R$. 
Nevertheless, $\left(\vec{b}_{n}, \vec{b}_{0}\right) \notin R \circ^{n} R$. As a matter of fact, if we assume otherwise, there exist $\vec{b}_{1}, \ldots, \vec{b}_{n-1} \in B$, where $\vec{b}_{i}:=\left(b_{i, 1}, \ldots, b_{i, n}\right)$, such that, for $1 \leq i \leq n,\left(\vec{b}_{i}, \vec{b}_{i-1}\right) \in R$. By expanding this latter relationship, we get the following:

$$
\vec{b}_{n}=\left[\begin{array}{c}
a_{2} \\
a_{3} \\
\vdots \\
a_{n} \\
a_{n+1}
\end{array}\right] R\left[\begin{array}{c}
b_{n-1,1} \\
b_{n-1,2} \\
\vdots \\
b_{n-1, n-1} \\
b_{n-1, n}
\end{array}\right] R \cdots R\left[\begin{array}{c}
b_{1,1} \\
b_{1,2} \\
\vdots \\
b_{1, n-1} \\
b_{1, n}
\end{array}\right] R\left[\begin{array}{c}
a_{0} \\
a_{1} \\
\vdots \\
a_{n-2} \\
a_{n-1}
\end{array}\right]=\vec{b}_{0}
$$

By looking at the components of the $n$-tuples $\vec{b}_{i}$ 's and how $R$ is defined, we have that

$$
\begin{aligned}
& a_{0} \alpha \circ \beta b_{1,1} ; \\
& b_{i, i} \beta b_{i, i+1} \beta \circ \alpha b_{i+1, i+1} \text { for odd } 1 \leq i \leq n-2 ; \\
& b_{i, i} \alpha b_{i, i+1} \alpha \circ \beta b_{i+1, i+1} \text { for even } 1 \leq i \leq n-2 ; \\
& b_{n-1, n-1} \beta b_{n-1, n} \beta \circ \alpha a_{n+1} \text { if } n \text { is even; } \\
& b_{n-1, n-1} \alpha b_{n-1, n} \alpha \circ \beta a_{n+1} \text { if } n \text { is odd. }
\end{aligned}
$$

Therefore, if $n$ is odd, the previous list yields

$$
a_{0} \alpha \circ \beta b_{1,1} \beta \circ \alpha \cdots b_{n-1, n-1} \alpha \circ \beta a_{n+1} ;
$$

while for $n$ even,

$$
a_{0} \alpha \circ \beta b_{1,1} \beta \circ \alpha \cdots b_{n-1, n-1} \beta \circ \alpha a_{n+1} \text {. }
$$

In either case, $\left(a_{0}, a_{n}\right) \in(\alpha \circ \beta) \circ^{n}(\beta \circ \alpha)=\alpha \circ^{n+1} \beta$, contradicting the initial assumption. 
Besides providing a certain procedure to build a Hagemann relation, Theorem 3.1 also lowers the power of the non- $n$-permutable algebra that is needed to find the $(n-1)$-dimensional Hagemann relation itself. As a matter of fact, if $\mathbf{A}$ is a failure of $n$-permutability, then Hagemann and Mitschke's approach would consist of finding a Hagemann relation of dimension $n-1$ as a subuniverse of $\mathbf{F}_{\operatorname{HSP}(\mathbf{A})}^{2}(\{x, y\})$, namely of $\left(\mathbf{A}^{|A|^{2}}\right)^{2}$. On the other hand, our approach produces an $(n-1)$-dimensional Hagemann relation as a subuniverse of $\mathbf{A}^{n-1} \times \mathbf{A}^{n-1}$.

As far as the converse reasoning is concerned, we can also prove that, whenever A carries a Hagemann relation of dimension $n$, then a failure of congruence $(n+1)$-permutability can be primitively positively defined within $\operatorname{HSP}(\mathbf{A})$. We have realized, after we found out a proof of it, that the next theorem generalizes the argument implicitly exposed in the last few lines of Example 3.8 of [10].

Theorem 3.2. Let $n \geq 1, \mathbf{A}$ be an algebra and $R \leq \mathbf{A} \times \mathbf{A}$ be a Hagemann relation of dimension $n$. Let $\mathbf{S}$ be the subalgebra of $\mathbf{A}^{n+1}$ whose universe is $S=R \star^{n} R$.

If $E, O \subseteq n+1$ denote, respectively, the sets of even and odd numbers in $n+1$, and $\theta_{E}, \theta_{O}$ are defined as

$$
\begin{aligned}
\theta_{E} & :=\bigwedge_{i \in E} \operatorname{ker} \pi_{i \mid S}, \\
\theta_{O} & :=\bigwedge_{i \in O} \operatorname{ker} \pi_{i \mid S},
\end{aligned}
$$

then $\mathbf{S}$ is a failure of congruence $(n+1)$-permutability, where $\theta_{E}{ }^{\circ+1}$ $\theta_{O} \neq \theta_{O}{ }^{n+1} \theta_{E}$. 
Proof. Let $n \geq 1, \mathbf{A}, R \leq \mathbf{A} \times \mathbf{A}, S \leq \mathbf{A}^{n+1}, \theta_{E}, \theta_{O} \in \operatorname{Con}(\mathbf{S})$ as in the statement.

Since $R$ is an $n$-dimensional Hagemann relation, there exist $a_{0}, a_{n} \in A$, with $\left(a_{0}, a_{n}\right) \in R$ but $\left(a_{n}, a_{0}\right) \notin R \circ^{n} R$. Moreover, we remark that $\left(a_{0}, a_{0}\right),\left(a_{n}, a_{n}\right) \in R$, by reflexivity.

Define the $(n+1)$-tuple $\vec{s}_{i} \in A^{n+1}$, for $0 \leq i \leq n+1$ :

$$
\vec{s}_{i}=(\overbrace{a_{0}, \ldots, a_{0}}^{n+1-i}, \underbrace{a_{n}, \ldots, a_{n}}_{i}) .
$$

It is straightforward to notice that $\vec{s}_{0}, \ldots, \vec{s}_{n+1}$ are elements of $R \star \star^{n} R=S$.

Furthermore, for every $0 \leq i \leq n$,

$$
\begin{aligned}
& \text { if } n \text { is odd, then }\left\{\begin{array}{ll}
\vec{s}_{i} \theta_{E} & \vec{s}_{i+1} \\
\vec{s}_{i} \theta_{O} & \vec{s}_{i+1}
\end{array} \quad \text { if } i\right. \text { is even; } \\
& \text { if } n \text { is odd; }
\end{aligned}
$$

Expanding these relationships, we get that, for $n$ odd,

$$
\vec{s}_{0} \theta_{E} \vec{s}_{1} \theta_{O} \cdots \theta_{E} \vec{s}_{n} \theta_{O} \vec{s}_{n+1},
$$

with $n+1$ alternating occurrences of $\theta_{O}$ and $\theta_{E}$, yielding $\left(\vec{s}_{0}, \vec{s}_{n+1}\right) \in$ $\theta_{E} \circ^{n+1} \theta_{O}$.

On the other hand, for $n$ even,

$$
\vec{s}_{0} \theta_{O} \vec{s}_{1} \theta_{E} \cdots \theta_{E} \vec{s}_{n} \theta_{O} \vec{s}_{n+1},
$$

again with $n+1$ alternating occurrences of $\theta_{O}$ and $\theta_{E}$, giving as a result $\left(\vec{s}_{0}, \vec{s}_{n+1}\right) \in \theta_{O}{ }^{\circ+1} \theta_{E}$. 
The next step is to show that $\theta_{O}$ and $\theta_{E}$ do not $(n+1)$-permute. Assume first $n$ is odd. We have just proven $\left(\vec{s}_{0}, \vec{s}_{n+1}\right) \in \theta_{E}{ }^{n+1} \theta_{O}$, and hence it suffices to prove that $\left(\vec{s}_{0}, \vec{s}_{n+1}\right) \notin \theta_{O}{ }^{\circ}{ }^{n+1} \theta_{E}$. Suppose otherwise, namely, there exist $\vec{r}_{1}, \ldots, \vec{r}_{n} \in S$, such that

$$
\begin{aligned}
& \vec{s}_{0} \theta_{O} \vec{r}_{1} ; \\
& \vec{r}_{i} \theta_{E} \vec{r}_{i+1} \text { for odd } 1 \leq i \leq n-1 ; \\
& \vec{r}_{i} \theta_{O} \vec{r}_{i+1} \text { for even } 1 \leq i \leq n-1 ; \\
& \vec{r}_{n} \theta_{E} \vec{s}_{n+1} .
\end{aligned}
$$

For $1 \leq i \leq n$, say $\vec{r}_{i}=\left(r_{i, 0}, \ldots, r_{i, n}\right)$. The first of the above relationships yields that $r_{1, i}=a_{0}$, for $i \in O$, while from the fourth we deduce $r_{n, i}=a_{n}$, for $i \in E$. The other two, instead, mean

$$
\begin{gathered}
r_{i, j}=r_{i+1, j} \text { for odd } 1 \leq i \leq n-2, j \in E ; \\
r_{i, j}=r_{i+1, j} \text { for even } 1 \leq i \leq n-2, j \in O .
\end{gathered}
$$

Pictorially:

$$
\begin{gathered}
\vec{s}_{0}=\left[\begin{array}{c}
a_{0} \\
a_{0} \\
\vdots \\
a_{0} \\
a_{0}
\end{array}\right] \theta_{O}\left[\begin{array}{c}
r_{1,0} \\
a_{0} \\
\vdots \\
r_{1, n-1} \\
a_{0}
\end{array}\right] \theta_{E}\left[\begin{array}{c}
r_{1,0} \\
r_{2,1} \\
\vdots \\
r_{1, n-1} \\
r_{2, n}
\end{array}\right] \theta_{O} \ldots \\
\cdots \theta_{E}\left[\begin{array}{c}
r_{n-2,0} \\
r_{n-1,1} \\
\vdots \\
r_{n-2, n-1} \\
r_{n-1, n}
\end{array}\right] \theta_{O}\left[\begin{array}{c}
a_{n} \\
r_{n-1,1} \\
\vdots \\
a_{n} \\
r_{n-1, n}
\end{array}\right] \theta_{E}\left[\begin{array}{c}
a_{n} \\
a_{n} \\
\vdots \\
a_{n} \\
a_{n}
\end{array}\right]=\vec{s}_{n+1} .
\end{gathered}
$$


As a result, by definition of $S$, we have that

$$
\begin{aligned}
& a_{n} R r_{n-1,1} ; \\
& r_{n-i, i} R r_{n-i-1, i+1} \text { for } 1 \leq i \leq n-2 ; \\
& r_{1, n-1} R a_{0} .
\end{aligned}
$$

These imply $\left(a_{n}, a_{0}\right) \in R \circ^{n} R$, contradicting the initial hypothesis.

The case for $n$ even proceeds analogously.

Even for this case, we want to point out the fact that an algebra carrying an $n$-dimensional Hagemann relation need not be a failure of congruence $(n+1)$-permutability; the following is a trivial counterexample.

Example 3.1. Consider the variety of sets, Sets, and let 2 be the (only, up to isomorphism) algebra of Sets with two elements, i.e., $\mathbf{2}=\langle\{0,1\}, \varnothing\rangle$. Obviously, Sets is not congruence $n$-permutable for any $n \geq 2$. Moreover, $\mathbf{F}_{\mathcal{S e t s}}(\mathbf{2})=\mathbf{2}$, hence, by Theorem $2.1, R=\{(0,0),(0,1)$, $(1,1)\}=\operatorname{Sg}^{2 \times 2}(R) \leq \mathbf{2} \times \mathbf{2}$ is a Hagemann relation of dimension $n$, for every $n \geq 1$. Nonetheless, $\mathbf{2}$ is not a failure of congruence $n$-permutability, for any $n \geq 2$ (being congruence 2-permutable).

In the next section we are going to deal with some suitable classes of varieties that are somehow related to congruence $n$-permutability. Therefore, for fixed $n \geq 2$, we define $C P_{n}$ to be the class of congruence $n$-permutable varieties and $C P_{\omega}$ to be the class of congruence $k$-permutable varieties, for some $k \geq 2$ (i.e., $C P_{\omega}=\bigcup_{k \geq 2} C P_{k}$ ). Also, we will denote by $C P_{n}^{i d}$ and $C P_{\omega}^{i d}$ the classes of idempotent varieties in, respectively, $C P_{n}$ and $C P_{\omega}$. 


\section{Omission Classes of Special Hagemann Relations}

We are going to consider a family of Hagemann relations with a particular configuration, having found the initial inspiration in Lemma 2 of [2]. First, we provide the following definition.

Definition 4.1. Let $n \geq 1$ and $\mathrm{A}$ be an algebra with $R \leq \mathrm{A} \times \mathrm{A}$. We say that $R$ is an $n$-dimensional special Hagemann relation, or a special Hagemann relation of dimension $n$, if there exists a partition $\left\{A_{i}: 0 \leq i\right.$ $\leq n+1\}$ of $A$, such that

$$
R=\left[\left(A_{0} \cup A_{1}\right) \times A\right] \cup \bigcup_{i=2}^{n+1}\left[A_{i} \times\left(\bigcup_{j=i-1}^{n+1} A_{j}\right)\right]
$$

In this setting, we also say that the partition $\left\{A_{i}: 0 \leq i \leq n+1\right\}$ induces $R$, or analogous expressions.

It is straightforward to notice that Definition 4.1 actually defines an $n$-dimensional Hagemann relation for every $n \geq 1$ : as a matter of fact, since $A_{i} \times A_{i} \subseteq R$, for every $0 \leq i \leq n+1, R$ is reflexive; moreover, $A_{0} \times A_{n+1} \subseteq R$, but there is no way to $\left(R \circ^{n} R\right)$-connect any $a_{n+1} \in A_{n+1}$ to any $a_{0} \in A_{0}$. However, it is always possible to $\left(R \circ^{n+1} R\right)$-connect any two elements of $A$, i.e., $(a, b) \in R \circ^{n+1} R$, for every $a, b \in A$ (equivalently, $A^{2}=R \circ^{n+1} R$ ); this yields that no $n$-dimensional special Hagemann relation can be an $(n+1)$-dimensional Hagemann relation, for any $n \geq 1$.

A Cartesian representation of a special Hagemann relation of dimension $n$ (Figure 1) could help visualize the particular configuration of such an object. 


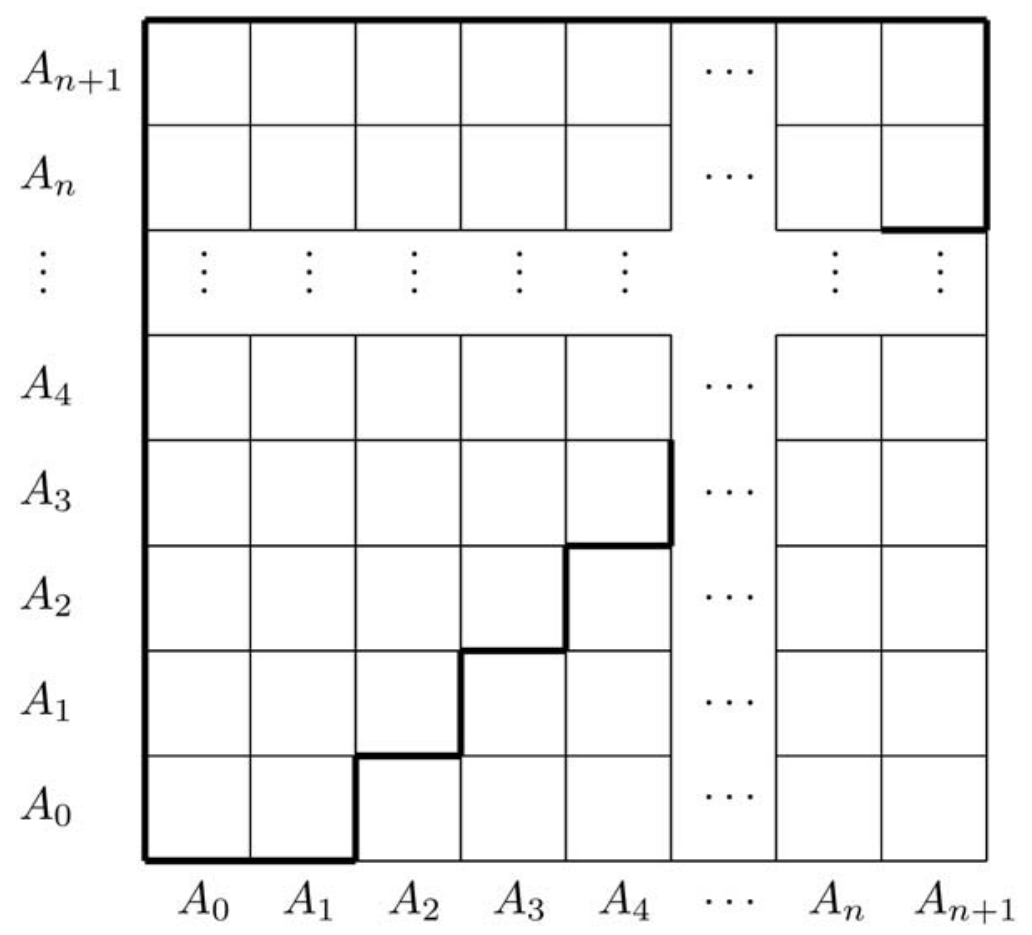

Figure 1. A Cartesian representation of an $n$-dimensional special Hagemann relation.

Let us consider those varieties in which no algebras carrying special Hagemann relations of some fixed dimension occur, as formally defined below.

Definition 4.2. Let $n \geq 1$ and $\mathcal{V}$ be a variety. We say that $\mathcal{V}$ admits $S H R_{n}$ if there exists an algebra $\mathbf{A} \in \mathcal{V}$ and $R \leq \mathbf{A}^{2}$, such that $R$ is an $n$-dimensional special Hagemann relation. On the other hand, we say that $\mathcal{V}$ omits $S H R_{n}$, whenever $\mathcal{V}$ does not admit $S H R_{n}$ and the class of varieties (up to equi-interpretability) that omit $S H R_{n}$ is denoted by $\Omega\left(S H R_{n}\right)$. Moreover, the subclass of $\Omega\left(S H R_{n}\right)$ containing all and only the idempotent varieties is denoted by $\Omega^{i d}\left(S H R_{n}\right)$. 
By Theorem 2.1, we can easily deduce that $C P_{n} \subseteq \Omega\left(S H R_{n-1}\right)$, for every $n \geq 2$. On the other hand, we have not been able to generally establish what kind of relationship occurs between $\Omega\left(S H R_{n}\right)$ and $\Omega\left(S H R_{m}\right)$, for distinct values of $m, n \geq 1$, although something has been figured out in the idempotent setting. In addition, we are able to prove the following.

Theorem 4.1. Let $n \geq 1$ and $\mathcal{V}$ be a variety. If $\mathcal{V}$ admits $S H R_{n}$, then $\mathcal{V}$ admits $S H R_{1}$ as well. In other words, for every $n \geq 1$,

$$
\Omega\left(S H R_{1}\right) \subseteq \Omega\left(S H R_{n}\right) .
$$

Proof. Fix $n \geq 1$ and let $\mathcal{V}$ be a variety admitting $S H R_{n}$. That is to say there exists $\mathbf{A} \in \mathcal{V}$ carrying a special Hagemann relation of dimension $n$; let us call it $R$ and assume that it is induced by the partition $\left\{A_{i}: 0 \leq i \leq n+1\right\}$ of $A$. We aim to show that the relation $R{ }^{n} R$ is a special Hagemann relation of dimension 1 on the algebra A. To do so, first notice that $R \circ^{n} R$ is still a subuniverse of $\mathrm{A}^{2}$; moreover, we have previously noted that $(x, y) \notin R{ }^{n} R$, for any $x \in A_{n+1}, y \in A_{0}$, showing that $A_{n+1} \times A_{0} \subseteq A^{2}-\left(R \circ^{n} R\right)$. If we call $A_{0}^{\prime}:=A_{0}, A_{1}^{\prime}:=\bigcup_{i=1}^{n} A_{i}$, $A_{2}^{\prime}:=A_{n+1}$, we have just proven that $A_{2}^{\prime} \times A_{0}^{\prime}$ is totally contained in the complement of $R{ }^{n} R$. On the other hand, it is straightforward to check that, for every $a_{i} \in A_{i}, 0 \leq i \leq n+1$, we have that $a_{n+1} R a_{n} R \cdots R a_{1} R a_{0}$. In particular, for all $0 \leq j \leq i \leq n$,

$$
a_{i+1} R a_{i} R \cdots R a_{j}
$$

with $i-j+1$ occurrences of $R$, proving that $A_{i+1} \times A_{j} \subseteq R \circ^{i-j+1} R$, which means

$$
A_{2}^{\prime} \times A_{1}^{\prime} \subseteq \bigcup_{i=1}^{n} R \circ^{n-i+1} R=R \circ^{n} R,
$$


and

$$
A_{1}^{\prime} \times A \subseteq \bigcup_{0 \leq j \leq i \leq n} R \circ^{i-j+1} R=R \circ^{n} R .
$$

Also $A_{2}^{\prime} \times A_{2}^{\prime}, A_{0}^{\prime} \times A \subseteq R \subseteq R{ }^{n} R$. This proves that the partition $\left\{A_{0}^{\prime}, A_{1}^{\prime}, A_{2}^{\prime}\right\}$ of $A$ induces $R \circ^{n} R$, which is hence a 1-dimensional special Hagemann relation.

As far as idempotent varieties are concerned, in that case we can prove what we consider an optimal framework.

Theorem 4.2. Let $n \geq 1$ and $\mathcal{V}$ be an idempotent variety. If $\mathcal{V}$ admits $S H R_{n+1}$, then $\mathcal{V}$ also admits $S H R_{n}$. In other words,

$$
\Omega^{i d}\left(S H R_{n}\right) \subseteq \Omega^{i d}\left(S H R_{n+1}\right) .
$$

Proof. Let $n \geq 1$ be fixed, along with an idempotent variety $\mathcal{V}$. Suppose $\mathcal{V}$ contains an algebra $\mathbf{A}$ that carries a special Hagemann relation $R$ of dimension $n+1$, induced by the partition $\left\{A_{i}: 0 \leq i \leq n+2\right\}$ of $A$. Let $B$ be the subset of $A$ defined by

$$
B=\bigcup_{i=1}^{n+2} A_{i}
$$

Equivalently, $B=A-A_{0}$. We claim that $B$ is a subuniverse of $\mathbf{A}$. As a matter of fact, if $b$ is any element of $A$ not belonging to $A_{0}$ and $a_{n+2}$ is any element in $A_{n+2}$, then $b \in A_{i}$, for some $i \in\{1, \ldots, n+2\}$, i.e., $a_{n+2}$ $R \circ^{n+2-i} R b$, if $i<n+2$, and $a_{n+2} R b$ otherwise. In either case, $a_{n+2}$ $R \circ^{n+1} R b$. Conversely, for any $a_{n+2} \in A_{n+2}$, if $b$ is any element of $A$ satisfying $a_{n+2} R \circ^{n+1} R b$, then $b \notin A_{0}$, otherwise contradicting that $R$ is a special Hagemann relation of dimension $n+1$. Therefore, we deduce that $B=a_{n+2} /\left(R \circ^{n+1} R\right)$, for any $a_{n+2} \in A_{n+2}$, implying our claim, by idempotence. 
At this point, if we call $S:=R \cap B^{2}$ and $B_{i}:=A_{i+1}$, for $0 \leq i \leq n+1$, an easy set theoretical computation yields that $S \leq \mathbf{B} \times \mathbf{B}$ is a special Hagemann relation of dimension $\mathrm{n}$, induced by the partition $\left\{B_{i}: 0 \leq i \leq n+1\right\}$ of $B$. Because $\mathrm{B} \in \mathcal{V}$, we get that $\mathcal{V}$ admits $S H R_{n}$, as desired.

In fact, the omission for a variety of $S H R_{n}$, for $n \geq 1$, is equivalent to satisfying a Maltsev condition, as the next theorem states.

Theorem 4.3. For $n \geq 1$, the omission class $\Omega\left(S H R_{n}\right)$ is a Maltsev class.

Proof. The proof of this fact will make use of Theorem 1.1 (see also [18]). We could have alternatively used another sufficient condition exposed in [17], but we prefer to explicitly show the details using Taylor's approach. Immediately notice that, for any $n \geq 1, \Omega\left(S H R_{n}\right) \neq \varnothing, C P_{n}$ being, in fact, contained in it. Thus, we need to verify that the four requirements of Theorem 1.1 are met, which we will refer to as (C1), (C2), (C3) and (C4).

Let us fix $n \geq 1$ and notice that (C1) and (C2) are obviously satisfied. The trickiest part of the entire proof is the verification of (C3), for which it suffices to consider the meet of any two varieties $\mathcal{V}$ and $\mathcal{W}$.

By contrapositive argument, assume $\mathcal{V} \wedge \mathcal{W} \notin \Omega\left(S H R_{n}\right)$. By definition of $\Omega\left(S H R_{n}\right)$, there must exist an algebra $\mathrm{Q} \in \mathcal{V} \wedge \mathcal{W}$ and a subuniverse $R \leq \mathbf{Q} \times \mathbf{Q}$ which is a special Hagemann relation of dimension $n$. Let $\left\{Q_{0}, \ldots, Q_{n+1}\right\}$ be the partition of $Q$ inducing $R$. 
Therefore, there exist $\mathrm{U} \in \mathcal{V}$ and $\mathrm{V} \in \mathcal{W}$, such that $\mathrm{Q} \cong \mathrm{U} \otimes \mathrm{V}$ and

$$
R \leq \mathrm{Q}^{2} \cong(\mathrm{U} \otimes \mathrm{V})^{2} \cong \mathrm{U}^{2} \otimes \mathrm{V}^{2}
$$

This in turns yields that there exist $R_{1} \leq \mathrm{U} \times \mathrm{U}$ and $R_{2} \leq \mathrm{V} \times \mathrm{V}$ with $\mathrm{R} \cong \mathrm{R}_{1} \otimes \mathrm{R}_{2}$. Without loss of generality, we will assume $Q=U \times V$ and $R=R_{1} \otimes R_{2}$ (in the sense of Definition 3.1), allowing us to use the following notation, according to which:

$$
\left[\begin{array}{l}
u_{1} \\
v_{1}
\end{array}\right] R\left[\begin{array}{l}
u_{2} \\
v_{2}
\end{array}\right] \text { if and only if }\left(u_{1}, u_{2}\right) \in R_{1} \text { and }\left(v_{1}, v_{2}\right) \in R_{2} \text {. }
$$

In such a case, it is straightforward to check that both $R_{1}$ and $R_{2}$ are reflexive relations, due to the reflexivity of $R$. The crucial part of the proof consists of showing that either $R_{1}=U \times U$ or $R_{2}=V \times V$.

Suppose $\quad R_{2} \subsetneq V^{2} \quad$ and let $\quad\left(v, v^{\prime}\right) \in V^{2} \quad$ with $\quad\left(v, v^{\prime}\right) \notin R_{2}$. Furthermore, pick any $\left(u_{1}, u_{2}\right) \in U^{2}$ and consider the pair $\left[\begin{array}{c}u_{2} \\ v\end{array}\right]$.

If we assume $\left[\begin{array}{c}u_{2} \\ v\end{array}\right] R\left[\begin{array}{c}u_{2} \\ v^{\prime}\end{array}\right]$, then $\left(v, v^{\prime}\right) \in R_{2}$, which is a contradiction. This means that there exists at least one element of $Q$ to which $\left[\begin{array}{c}u_{2} \\ v\end{array}\right]$ cannot be $R$-related: since $\left(Q_{0} \cup Q_{1}\right) \times Q \subseteq R$, we deduce that $\left[\begin{array}{c}u_{2} \\ v\end{array}\right] \notin Q_{0} \cup Q_{1}$. Therefore, $\left[\begin{array}{c}u_{2} \\ v\end{array}\right] \in \bigcup_{i=2}^{n+1} Q_{i}$.

Then, suppose that $\left[\begin{array}{c}u_{2} \\ v\end{array}\right] \in Q_{i}$, for some $2 \leq i \leq n+1$ and consider the pair $\left[\begin{array}{c}u_{1} \\ v^{\prime}\end{array}\right]$. If it is the case that $\left[\begin{array}{c}u_{1} \\ v^{\prime}\end{array}\right] \in \bigcup_{j=i-1}^{n+1} Q_{j}$, then, by the fact that $Q_{i} \times\left(Q_{i-1} \cup \ldots \cup Q_{n+1}\right) \subseteq R$, we would have that $\left[\begin{array}{c}u_{2} \\ v\end{array}\right] R\left[\begin{array}{c}u_{1} \\ v^{\prime}\end{array}\right]$, proving $\left(v, v^{\prime}\right) \in R_{2}$ and thus contradicting the initial assumption on this pair. 
Hence, it follows that

$$
\left[\begin{array}{c}
u_{1} \\
v^{\prime}
\end{array}\right] \in \bigcup_{j=0}^{i-2} Q_{j}
$$

and because $Q_{j} \times Q_{i} \subseteq R$, for all $0 \leq j \leq i-2$, then

$$
\left[\begin{array}{c}
u_{1} \\
v^{\prime}
\end{array}\right] R\left[\begin{array}{c}
u_{2} \\
v
\end{array}\right],
$$

proving that $\left(u_{1}, u_{2}\right) \in R_{1}$. Since this pair was arbitrary in $U^{2}$, this shows that $R_{1}=U^{2}$, as desired.

Symmetrically, we can show that, if $R_{1} \subsetneq U^{2}$, then $R_{2}=V^{2}$.

Henceforth, we can assume, without any loss of generality, that $R=R_{1} \otimes V^{2} \quad$ (the symmetric case $R=U^{2} \otimes R_{2}$ would proceed analogously). If $\pi: Q \rightarrow U$ is the projection map onto $U$, then call $U_{i}:=\pi\left(Q_{i}\right)$ for all $0 \leq i \leq n+1$. We show that, for every $0 \leq i \leq n+1, U_{i}$ is non-empty (obvious, since $Q_{i} \neq \varnothing$ ) and, furthermore, $\bigcup_{i=0}^{n+1} U_{i}=U$

As a matter of fact, if $u \in U$, then for any $v \in V$ the pair $\left[\begin{array}{l}u \\ v\end{array}\right]$ lies in $Q$, and hence in $Q_{i}$, for some $0 \leq i \leq n+1$, yielding $u=\pi\left(\left[\begin{array}{l}u \\ v\end{array}\right]\right) \in \pi\left(Q_{i}\right)=U_{i}$.

Moreover, for $0 \leq i, j \leq n+1$, with $i \neq j, U_{i} \cap U_{j}=\varnothing$. It is sufficient to prove that, for any fixed $0 \leq i<n+1$ and $i<j \leq n+1$, $U_{i} \cap U_{j}=\varnothing$. 
Let us begin with $0 \leq i \leq n-1$ and $i<j \leq n+1$ : suppose $U_{i} \cap U_{j} \neq \varnothing$, and let $u \in U_{i} \cap U_{j}$. Thus, by definition of the $U_{i}$ 's, there exist $v, v^{\prime} \in V$ such that $\left[\begin{array}{l}u \\ v\end{array}\right] \in Q_{i}$ and $\left[\begin{array}{c}u \\ v^{\prime}\end{array}\right] \in Q_{j}$. Because $j>i, Q_{i+2} \times Q_{j} \subseteq R$, which implies the existence of some $\left[\begin{array}{l}x \\ y\end{array}\right] \in Q_{i+2}$ such that

$$
\left[\begin{array}{l}
x \\
y
\end{array}\right] R\left[\begin{array}{l}
u \\
v^{\prime}
\end{array}\right] .
$$

This proves that $(x, u) \in R_{1}$ and, since $R=R_{1} \otimes V^{2}$, we deduce that $\left[\begin{array}{l}x \\ y\end{array}\right] R\left[\begin{array}{l}u \\ v\end{array}\right]$, contradicting the fact that $\left(Q_{i+2} \times Q_{i}\right) \cap R=\varnothing$. The contradiction derives from not assuming $U_{i} \cap U_{j}=\varnothing$, which instead holds.

The only remaining case is when $i=n$ and $j=n+1$. Therefore, suppose $U_{n} \cap U_{n+1}$ contains an element $u$ and, again, let $v, v^{\prime}$ be elements of $V$ such that $\left[\begin{array}{l}u \\ v\end{array}\right] \in Q_{n}$ and $\left[\begin{array}{l}u \\ v^{\prime}\end{array}\right] \in Q_{n+1}$. Since $Q_{n} \times Q_{n-1} \subseteq R$, let $\left[\begin{array}{l}x \\ y\end{array}\right] \in Q_{n-1}$ with

$$
\left[\begin{array}{l}
u \\
v
\end{array}\right] R\left[\begin{array}{l}
x \\
y
\end{array}\right],
$$

which also means $(u, x) \in R_{1}$. Thus, since $R=R_{1} \otimes V^{2}$, we also have that

$$
\left[\begin{array}{l}
u \\
v^{\prime}
\end{array}\right] R\left[\begin{array}{l}
x \\
y
\end{array}\right],
$$

contradicting the fact that $\left(Q_{n+1} \times Q_{n-1}\right) \cap R=\varnothing$. Again, the contradiction comes from not having considered $U_{n} \cap U_{n+1}$ empty, which indeed is. 
Finally, we prove that $R_{1}$ has the desired configuration, i.e.,

$$
R_{1}=\left[\left(U_{0} \cup U_{1}\right) \times U\right] \cup \bigcup_{i=2}^{n+1}\left[U_{i} \times\left(U_{i-1} \cup \ldots \cup U_{n+1}\right)\right]
$$

Call $T$ the right-hand-side of the equality displayed above.

If $\left(u, u^{\prime}\right) \in R_{1}$, then there exist $v, v^{\prime} \in V$ such that $\left[\begin{array}{l}u \\ v\end{array}\right] R\left[\begin{array}{l}u^{\prime} \\ v^{\prime}\end{array}\right]$, which means that $\left[\begin{array}{l}u \\ v\end{array}\right] \in Q_{0}$, or $\left[\begin{array}{l}u \\ v\end{array}\right] \in Q_{i}$ and $\left[\begin{array}{l}u^{\prime} \\ v^{\prime}\end{array}\right] \in Q_{j}$, for some $1 \leq i \leq n+1, i-1 \leq j \leq n+1$. Hence, either $u \in U_{0}$, or $u \in U_{i}$ and $u^{\prime} \in U_{j}$, proving that $\left(u, u^{\prime}\right) \in T$. By inverting the above reasoning, we prove the other inclusion as well and hence that the equality holds.

These properties show that $\left\{U_{i}: 0 \leq i \leq n+1\right\}$ is a partition of $U$ and such a partition induces $R_{1}$, which is an $n$-dimensional special Hagemann relation on the algebra $\mathbf{U}$. Since $\mathbf{U}$ is a member of $\mathcal{V}$, we have indeed proven that $\mathcal{V}$ admits $S H R_{n}$, as desired.

In order to prove $(\mathrm{C} 4)$, we are going to invoke the Compactness Theorem while arguing again by contrapositive. Thus, suppose $\sum$ is a set of equations of type $\sigma$ such that, for every finite $\Psi \subseteq \sum, \operatorname{Mod}(\Psi) \notin$ $\Omega\left(S H R_{n}\right)$ [more precisely and formally, think of $\sum$ as a set of universally quantified sentences of the form $\forall x_{1} \ldots \forall x_{k}\left(p\left(x_{1}, \ldots, x_{k}\right) \approx\right.$ $\left.\left.q\left(x_{1}, \ldots, x_{k}\right)\right)\right]$. We aim to prove that also $\operatorname{Mod}\left(\sum\right) \notin \Omega\left(S H R_{n}\right)$.

Hence, call $F$ the set of fundamental operation symbols of $\operatorname{Mod}\left(\sum\right)$ of type $\sigma$, and define the first order language $\mathcal{L}$ as follows:

$$
\mathcal{L}=F \cup\{R\} \bigcup\left\{A_{i}: 0 \leq i \leq n+1\right\},
$$


where each $A_{i}$ is a unary predicate and $R$ is binary, instead. Furthermore, let $\Gamma$ be the set of first order sentences listed below (the first sentence is indeed a scheme of axioms as $f$ varies in $F$ and $k$ is the arity of $f$ :

$$
\begin{gathered}
\forall x_{1} \ldots \forall x_{k} \forall y_{1} \ldots \forall y_{k}\left[\left(\bigwedge_{i=1}^{k} R\left(x_{i}, y_{i}\right)\right] \rightarrow R\left(f\left(x_{1}, \ldots, x_{k}\right), f\left(y_{1}, \ldots, y_{k}\right)\right)\right] ; \\
\forall x\left[\bigvee_{i=0}^{n+1} A_{i}(x)\right] ; \\
\exists x A_{i}(x) \text { for } i \in\{0, \ldots, n+1\} ; \\
\forall x\left[A_{i}(x) \rightarrow \neg A_{j}(x)\right] \text { for } 0 \leq i, j \leq n+1, i \neq j ; \\
\forall x \forall y\left[R(x, y) \leftrightarrow\left(A_{0}(x) \vee A_{1}(x) \vee \bigvee_{i=2}^{n+1}\left(A_{i}(x) \rightarrow \bigwedge_{j=0}^{i-2} \neg A_{j}(y)\right)\right)\right] .
\end{gathered}
$$

At this point, let $\Delta$ be any finite subset of $\sum \cup \Gamma$. We will show that $\Delta$ has a model.

Let $\Psi$ be any finite subset of $\sum$ : by assumption $\operatorname{Mod}(\Psi)$ admits $S H R_{n}$, i.e., there exists an algebra $\mathbf{A}=\left\langle A ;\left\{f^{\mathbf{A}}: f \in F\right\}\right\rangle \in \operatorname{Mod}(\Psi)$ such that $R^{\prime} \leq \mathbf{A} \times \mathbf{A}$ is a special Hagemann relation of dimension $n$. Let $\left\{A_{0}^{\prime}, \ldots, A_{n+1}^{\prime}\right\}$ be the partition inducing $R^{\prime}$. If we interpret the predicates of $\mathcal{L}$ in the natural way, namely, for all $x, y \in A, 0 \leq i \leq n+1$,

$$
\begin{aligned}
R^{\mathfrak{A}}(x, y) & \Leftrightarrow(x, y) \in R^{\prime}, \\
A_{i}^{\mathfrak{A}}(x) & \Leftrightarrow x \in A_{i}^{\prime},
\end{aligned}
$$

then the structure $\mathfrak{A}=\left\langle\mathbf{A} ; R^{\mathfrak{A}}, A_{0}^{\mathfrak{A}}, \ldots, A_{n+1}^{\mathfrak{A}}\right\rangle$ is a model of $\Delta$ by construction. 
Therefore, by the compactness theorem, we deduce that also $\sum \cup \Gamma$ has a model; if we only consider the universe of such a model along with the function symbols of $F$, we get an algebra $\mathbf{A}$ carrying a special Hagemann relation of dimension $n$, which is a model of $\sum$, proving that $\operatorname{Mod}\left(\sum\right) \notin \Omega\left(S H R_{n}\right)$, as we wanted to prove.

Unfortunately, we ignore the Maltsev condition characterizing $\Omega\left(S H R_{n}\right)$, which must exist by the above theorem, nor do we know whether such a Maltsev condition may be strong. In spite of this, we can rather directly deduce the following corollary of Theorem 4.3, also given the particular shape of a special Hagemann relation of dimension $n$.

Corollary 4.3.1. For every $n \geq 1, \Omega\left(S H R_{n}\right)$ is a prime Maltsev class.

Proof. Fix $n \geq 1$ and assume that $\mathcal{V}$ and $\mathcal{W}$ are two varieties admitting $S H R_{n}$. Thus, let $R \leq \mathrm{A}^{2}$ and $S \leq \mathrm{B}^{2}$ be special Hagemann relations of dimension $n$, induced by the respective partitions $\left\{A_{i}: 0 \leq\right.$ $i \leq n+1\}$ and $\left\{B_{i}: 0 \leq i \leq n+1\right\}$ of, respectively, $A$ and $B$, where $\mathbf{A} \in \mathcal{V}$ and $\mathbf{B} \in \mathcal{W}$. Let $\kappa>0$ be a properly chosen cardinal, so that $\left|A_{i} \times A^{\kappa}\right|=\left|B_{i} \times B^{\kappa}\right|$, for all $0 \leq i \leq n+1$. Denote such sets as $A_{i}^{\prime}:=A_{i}$ $\times A^{\kappa}, B_{i}^{\prime}:=B_{i} \times B^{\kappa}$, for $0 \leq i \leq n+1$, and further call $A^{\prime}:=A \times A^{\kappa}$ and $B^{\prime}:=B \times B^{\kappa}, R^{\prime}:=R \otimes\left(A^{\kappa}\right)^{2}$ and $S^{\prime}:=S \otimes\left(B^{\kappa}\right)^{2}$. It is obvious that $\left|A^{\prime}\right|=\left|B^{\prime}\right|,\left|R^{\prime}\right|=\left|S^{\prime}\right|$. Moreover, it is not hard to check that $R^{\prime} \leq \mathrm{A}^{\prime} \times \mathrm{A}^{\prime}$ and $S^{\prime} \leq \mathrm{B}^{\prime} \times \mathrm{B}^{\prime}$ are still $n$-dimensional special Hagemann relations, induced by, respectively, $\left\{A_{i}^{\prime}: 0 \leq i \leq n+1\right\}$ and $\left\{B_{i}^{\prime}: 0 \leq i \leq n+1\right\}$. This allows us to build a suitable algebra $\mathbf{C} \in \mathcal{V} \vee \mathcal{W}$, with $C=A^{\prime}$ and such that $R^{\prime} \leq \mathrm{C}^{2}$ is a special Hagemann relation of dimension $n$, showing that $\mathcal{V} \vee \mathcal{W}$ also admits $S H R_{n}$, yielding the primeness of $\Omega\left(S H R_{n}\right)$. 
Corollary 4.3.1 necessarily implies that $\Omega\left(S H R_{n}\right)$ is prime with respect to idem-potent varieties as well, for each $n \geq 1$. In particular, we are going to show that this fact also yields the primeness for some cases of congruence $n$-permutability in the idempotent setting. The first case we deal with concerns the strong Maltsev condition of congruence 2-permutability, the primeness of which has been discussed for years, ever since Garcia and Taylor conjectured it in [5]. In fact, nearly at the same time, Tschantz verified such a conjecture, although his later preprint paper ([20]) has never been published; indeed, his proof contains highly complex syntactic techniques that have had the universal algebraists community wonder whether there could exist a simpler semantic approach to the conjecture. It has turned out that, as far as idempotent varieties are concerned, some semantic primeness arguments can be developed, as witnessed in [15] and [8], where the authors verify the property by considering congruence 2-permutability as coincident with the strong Maltsev condition of "having a 2-cube term" (for more details on cube terms, refer to [1]). As one can expect, we characterize the class of idempotent congruence 2-permutable varieties via the omission of $S H R_{1}$. A preliminary discussion is however needed before we state the result.

Let us define a new object which we refer to as a special failure of congruence 2-permutability.

Definition 4.3. Let $\mathbf{A}$ and $\mathbf{B}$ be two similar algebras and $\mathbf{S} \leq \mathrm{A} \times \mathrm{B}$. We say that $\mathbf{S}$ is a special failure of congruence 2-permutability if there exist non-empty $P \subsetneq A$ and $Q \subsetneq B$ such that $S=(P \times B) \cup(A \times Q)$.

Likewise for the admission and omission of $S H R_{n}$, we define the omission class $\Omega\left(S F_{2}\right)$ as the class of all varieties containing no special failure of congruence 2-permutability, in which case we say that they omit $\mathrm{SF}_{2}$. It is straightforward to show that a special failure of 
congruence 2-permutability is a non-congruence 2-permutable algebra; this means that $C P_{2} \subseteq \Omega\left(S F_{2}\right)$. It is actually the case that the omission classes of $S H R_{1}$ and $S F_{2}$ coincide.

Lemma 4.1. For a variety $\mathcal{V}, \mathcal{V}$ omits $\mathrm{SF}_{2}$ if and only if it omits $S H R_{1}$. In other words, $\Omega\left(S H R_{1}\right)=\Omega\left(S F_{2}\right)$.

Proof. Let $\mathcal{V}$ be any variety throughout the proof. If $\mathcal{V}$ admits $S H R_{1}$, say $R \leq \mathrm{A}^{2}$, for $\mathrm{A} \in \mathcal{V}$, induced by $\left\{A_{0}, A_{1}, A_{2}\right\}$, then it is not hard to prove that $\mathrm{R}$ itself is a special failure of congruence 2-permutability, with $P=A_{0} \cup A_{1}$ and $Q=A_{1} \cup A_{2}$.

Conversely, if $\mathbf{S} \in \mathcal{V}$ is a special failure of congruence 2-permutability, with $\mathrm{S}, \mathrm{A}, \mathrm{B}, P$ and $Q$ as defined in Definition 4.3, then we can find an algebra in $\mathcal{V}$ carrying a 1-dimensional special Hagemann relation. Such an algebra is exactly $\mathbf{S}$ : as a matter of fact, if we define

$$
\begin{aligned}
S_{0} & :=P \times(B-Q), \\
S_{1} & :=P \times Q, \\
S_{2} & :=(A-P) \times Q,
\end{aligned}
$$

then it is a standard computation to verify that $\left\{S_{0}, S_{1}, S_{2}\right\}$ is a partition of $S$. Moreover, if $\alpha$ and $\beta$ denote the restrictions to $S$ of the kernels of the projection maps from $A \times B$ onto, respectively, $A$ and $B$, then the relation $R:=\alpha \circ \beta$ is a subuniverse of $\mathbf{S}^{2}$. We aim to show that $R$ is a special Hagemann relation of dimension 1 induced by $\left\{S_{0}, S_{1}, S_{2}\right\}$.

Assume first $(a, b) \in S_{i}$, for $i \in\{0,1\}$, which in particular yields $a \in P$. Then, for any $(x, y) \in S$, we have that

$$
\left[\begin{array}{l}
a \\
b
\end{array}\right] \alpha\left[\begin{array}{l}
\alpha \\
y
\end{array}\right] \beta\left[\begin{array}{l}
x \\
y
\end{array}\right],
$$

because $(a, y) \in P \times B \subseteq S$. This shows that $(a, b) R(x, y)$ and hence $\left(S_{0} \cup S_{1}\right) \times S \subseteq R$ 
On the other hand, if $(a, b) \in S_{2}$, then $a \notin P$ and $b \in Q$. Therefore, for every $(x, y) \in S$, if $(a, b) R(x, y)$, then we deduce that $(a, y) \in S$, forcing $y \in Q$ (since $a \notin P$ ) and hence $(x, y) \in S_{1} \cup S_{2}$. Conversely, for every $(x, y) \in S_{1} \cup S_{2}$, we have that $y \in Q$, which forces $(a, y) \in S_{2}$ and hence

$$
\left[\begin{array}{l}
a \\
b
\end{array}\right] \alpha\left[\begin{array}{l}
a \\
y
\end{array}\right] \beta\left[\begin{array}{l}
x \\
y
\end{array}\right],
$$

or equivalently $(a, b) R(x, y)$. This shows that $\left(S_{2} \times S_{0}\right) \cap R=\varnothing$ and $S_{2} \times\left(S_{1} \cup S_{2}\right) \subseteq R$, completing the proof.

Notice that this lemma does not require the assumption of idempotence. Next, we make use of the above property to prove the following criterion.

Theorem 4.4. For any idempotent variety $\mathcal{V}$, the following are equivalent:

(1) $\mathcal{V}$ is congruence 2-permutable;

(2) $\mathcal{V}$ omits $S H R_{1}$.

As a result, $C P_{2}^{i d}=\Omega^{i d}\left(S H R_{1}\right)$, implying that $C P_{2}$ is prime with respect to idempotent varieties.

Proof. Let $\mathcal{V}$ be any idempotent variety. If $\mathcal{V}$ is 2-permutable, then it must also omit $S H R_{1}$ by Theorem 2.1, proving that (1) implies (2).

Conversely, suppose that $\mathcal{V}$ is not congruence 2-permutable. By Theorem 2.1, the relation $R=S g \mathrm{~F}^{2}(\{(x, x),(x, y),(y, y)\})$ is a Hagemann relation of dimension 1 (caveat: we are not claiming that it is special), where $\mathrm{F}$ is the free algebra of $\mathcal{V}$ generated by $\{x, y\}$. At this point, we invoke Lemma 2.8 of [9], where the authors essentially show that $\mathbf{R}$ can be extended to a subalgebra $\mathbf{S} \leq \mathbf{F} \times \mathbf{F}$, which, according to 
our notation, turns out to be a special failure of congruence 2-permutability. Therefore, since $\mathcal{V}$ admits $S F_{2}$, by Lemma 4.1, $\mathcal{V}$ also admits $S H R_{1}$, showing that (2) implies (1) as well. Finally, the primeness property follows from Corollary 4.3.1.

Theorem 4.4 also shows that $\Omega^{i d}\left(S H R_{1}\right)$ is characterized by a strong Maltsev condition, since it coincides with $C P_{2}^{i d}$.

The second topic we wish to discuss is related to the case of congruence 3-permutability. As one could expect, a natural generalization of the result of Theorem 4.4 to (idempotent) congruence 3 -permutable varieties could be represented by a characterization via the omission of $\mathrm{SHR}_{2}$. Nonetheless, such an expectation turns out to be wrong, because we can prove that $C P_{3}^{i d} \subsetneq \Omega^{i d}\left(S H R_{2}\right)$. On the other hand, it is possible to characterize the class of locally finite idempotent congruence 3-permutable varieties via the omission of a slightly modified version of a special Hagemann relation of dimension 2, which we have called a 2-dimensional special Hagemann relation with middle part; this result provides, in fact, a primeness argument for congruence 3-permutability with respect to locally finite idempotent varieties. If one wants to look over the details of such themes, they are thoroughly discussed in [4] and [3].

The last case we focus on is the Maltsev condition of "congruence $n$-permutability, for some $n \geq 2$ ", or, equivalently, the Maltsev class $C P_{\omega}$. In particular, we are interested in the class $C P_{\omega}^{i d}$. A result of Valeriote and Willard in [21] (generalizing a previous result of Hobby and McKenzie of [7], holding for locally finite idempotent varieties) is that an idempotent variety $\mathcal{V}$ is congruence $n$-permutable, for some $n \geq 2$, if and only if $\mathcal{V} \leq \mathcal{D}$, where $\mathcal{D}$ denotes the variety of distributive lattices. We rephrase such a result as follows. 
Theorem 4.5 ([21]). If $\mathcal{D}$ denotes the variety of distributive lattices, then

$$
C P_{\omega}^{i d}=\left\{\mathcal{V} \in L^{i d}: \mathcal{V} \leq \mathcal{D}\right\}
$$

By using this theorem, we can prove the characterization below, where we set $\Omega^{i d}\left(S H R_{\omega}\right):=\bigcup_{n \geq 1} \Omega^{i d}\left(S H R_{n}\right)$.

Theorem 4.6. For any idempotent variety $\mathcal{V}, \mathcal{V}$ is congruence $n$-permutable, for some $n \geq 2$, if and only if it omits $S H R_{m}$, for some $m \geq 1$.

In other words, the following identity holds:

$$
C P_{\omega}^{i d}=\Omega^{i d}\left(S H R_{\omega}\right) .
$$

Proof. Let $\mathcal{V}$ be any idempotent variety for the rest of the proof. If $\mathcal{V}$ admits $S H R_{m}$, for every $m \geq 1$, then, by Theorem 2.1, it cannot be congruence $(m+1)$-permutable either, proving that $C P_{\omega}^{i d} \subseteq \Omega^{i d}\left(S H R_{\omega}\right)$.

Conversely, suppose that $\mathcal{V}$ is not congruence $n$-permutable, for any $n \geq 2$. Then, Theorem 4.5 guarantees that $\mathcal{V} \leq \mathcal{D}$, which is to say that $\mathcal{V}$ contains an algebra which is term equivalent to a distributive lattice $\mathrm{D}$, for every $\mathbf{D} \in \mathcal{D}$. In particular, $\mathcal{V}$ contains an algebra which is term equivalent to the $n$-chain $\mathbf{C}_{n}$, for every $n \geq 1$. Without loss of generality, we may assume that $\mathbf{C}_{n} \in \mathcal{V}$, for every $n \geq 1$. Recall that the $n$-chain is the lattice $\langle\{0, \ldots, n-1\} ; \vee, \wedge\rangle$, where $x \vee y=\max \{x, y\}$ and $x \wedge y=\min \{x, y\}$.

Fix $n \geq 3$ and define on $C_{n}=\{0, \ldots, n-1\}$ the relation $R_{n}$, as

$$
R_{n}=\left(\{0\} \times C_{n}\right) \cup \bigcup_{i=1}^{n-1}(\{i\} \times\{i-1, \ldots, n-1\}) .
$$


We show that $R_{n} \leq \mathrm{C}_{n}^{2}$. For convenience, call $X:=\{0\} \times C_{n}$ and $Y:=\bigcup_{i=1}^{n-1}(\{i\} \times\{i-1, \ldots, n-1\})$, so that $R_{n}=X \cup Y$. If $(0, k),(0, h) \in X$, then

$$
(0, k) \wedge(0, h)=(0, k \wedge h)
$$

which is an element of $X$ and then of $R_{n}$. If $(0, k) \in X$ and $(i, j) \in Y$, then

$$
(0, k) \wedge(i, j)=(0 \wedge i, k \wedge j)=(0, k \wedge j) \in X
$$

showing the closure also for this case. Finally, if $(i, j),(h, k) \in Y$, then necessarily $i-1 \leq j<n$ and $h-1 \leq k<n$, implying that $\min (j, k) \geq$ $\min \{i-1, h-1\}=\min \{i, h\}-1$ and hence showing that

$$
(i, j) \wedge(h, k)=(i \wedge h, j \wedge k)=(\min \{i, h\}, \min \{j, k\}) \in Y .
$$

A completely analogous dual reasoning (which we omit) yields the closure under $\vee$ as well, after noting that $R_{n}$ can also be represented as

$$
R_{n}=\left(C_{n} \times\{n-1\}\right) \cup \bigcup_{i=0}^{n-2}(\{0, \ldots, i+1\} \times\{i\})
$$

Thus, the relation $R_{n}$ is a subuniverse of $\mathrm{C}_{n}^{2}$ and, furthermore, it is induced by the partition $\{\{i\}: 0 \leq i \leq n-1\}$ of $C_{n}$ as a special Hagemann relation of dimension $n-2$. This shows that $\mathcal{V}$ admits $S H R_{m}$, for every $m \geq 1$, proving that $\Omega^{i d}\left(S H R_{\omega}\right) \subseteq C P_{\omega}^{i d}$, and hence the equality.

As a result, Theorem 4.6 yields that $C P_{\omega}$ is a prime Maltsev class with respect to idempotent varieties. In fact, such a primeness property was already derived from Theorem 4.5 , since $\mathcal{D}$ is a common upper bound for all idempotent non- $n$-permutable varieties, for any $n \geq 2$. Nonetheless, Theorem 4.6 emphasizes the fact that, for idempotent 
varieties, the failure of congruence $n$-permutability for every $n \geq 2$ is equivalent to the omission of very particular Hagemann relations of every dimension.

\section{The Non-Idempotent Setting}

This last section is meant to provide a justification to the fact that the class $\Omega\left(S H R_{n}\right)$ cannot coincide with the class $C P_{n+1}$, for any $n \geq 1$. What we have learnt so far is that $C P_{2}^{i d}=\Omega^{i d}\left(S H R_{1}\right)$ (Theorem 4.4) and $C P_{3}^{i d} \subseteq \Omega^{i d}\left(M^{+} S H R_{2}\right) \subsetneq \Omega^{i d}\left(S H R_{2}\right)$ (see [4] and [3]), meaning that for $n=3$, it is not possible to characterize the class of idempotent congruence 3-permutable varieties via the omission of $S H R_{2}$. Next, we are going to present an example of a non-idempotent variety that is not congruence $n$-permutable, for any $n \geq 2$, despite belonging to $\Omega\left(S H R_{k}\right)$, for every $k \geq 1$. This contribution has been provided by Dr. Agnes Szendrei, during the reviewing process of [4], as the external member of the Ph.D. examining committee. Before presenting her argument, we need to include a definition that can be found in Exercise 3.12(4) of [7].

Definition 5.1 ([7]). For $k \geq 1$ and an algebra $\mathbf{A}=\left\langle A ;\left\{f^{\mathbf{A}}: f \in F\right\}\right\rangle$, the $k$-th matrix power of $\mathbf{A}$ is the algebra $\mathbf{A}^{[k]}:=\left\langle A^{k} ;\left\{f^{\mathbf{A}^{k}}: f \in F\right\}\right.$ $\left.\cup\left\{d^{\mathrm{A}^{[k]}}, p^{\mathrm{A}^{[k]}}\right\}\right\rangle$, such that $d^{\mathrm{A}^{[k]}}$ is $k$-ary, $p^{\mathrm{A}^{[k]}}$ is unary and

$$
\begin{gathered}
d^{\mathbf{A}^{[k]}}\left(\left[\begin{array}{c}
x_{1}^{1} \\
\vdots \\
x_{k}^{1}
\end{array}\right], \ldots,\left[\begin{array}{c}
x_{1}^{k} \\
\vdots \\
x_{k}^{k}
\end{array}\right]\right)=\left[\begin{array}{c}
x_{1}^{1} \\
\vdots \\
x_{k}^{k}
\end{array}\right], \\
p^{\mathbf{A}^{[k]}}\left(\left(x_{1}, x_{2}, \ldots, x_{k}\right)\right)=\left(x_{2}, \ldots, x_{k}, x_{1}\right) .
\end{gathered}
$$

For a class $K$ of similar algebras, also define $K^{[k]}=\left\{\mathbf{A}^{[k]}: \mathbf{A} \in K\right\}$. 
It turns out that, whenever $\mathcal{V}$ is a variety, then $\mathcal{V}^{[k]}$ is a variety as well, for every $k \geq 1$. Moreover, a crucial property that we need in the next argument is that, whenever $R \leq \mathrm{A}^{[2]} \times \mathrm{A}^{[2]}$, then $R \cong E \otimes E$, for some $E \leq \mathrm{A}^{2}$.

That being said, in order to show that $C P_{n} \subsetneq \Omega\left(S H R_{n-1}\right)$, for all $n \geq 2$, it is sufficient to prove that $\mathcal{S e t s}^{[2]} \in \Omega\left(S H R_{1}\right)$ (and hence in $\Omega\left(S H R_{k}\right)$, for every $k \geq 1$, due to Theorem 4.1), although $\operatorname{Sets}^{[2]} \notin C P_{\omega}$ (also notice that $\operatorname{Sets}^{[2]}$ is not idempotent).

Dr. Szendrei's argument proceeds by first proving that $\mathcal{S e t s}^{[2]}$ is not congruence $n$-permutable for any $n \leq 2$, since it cannot realize any Hagemann-Mitschke terms. As a matter of fact, the only idempotent terms of $\mathcal{S e t s}^{[2]}$ are the projections and $d(x, y)$, for $x \neq y$, preventing the axiomatizing equations of $\mathcal{C P}$ from being satisfied, for every $n \geq 2$.

Furthermore, if $\mathcal{S e t s}^{[2]}$ admits $S H R_{1}$, then there exists a 1-dimensional special Hagemann relation $R \leq \mathrm{A}^{[2]} \times \mathrm{A}^{[2]}$, for some $\mathbf{A} \in$ Sets. In turn, because of the previous observation, there exists $E \leq \mathrm{A}^{2}$, such that $R \cong E \otimes E$. However, the proof of Theorem 4.3 ensures that $E=A^{2}$, and hence $R=A^{2} \times A^{2}$, contradicting its definition and showing that $\operatorname{Sets}^{[2]} \in \Omega\left(S H R_{k}\right)$, for every $k \geq 1$.

\section{References}

[1] J. Berman, P. Idziak, P. Markovic, R. McKenzie, M. Valeriote and R. Willard, Varieties with few subalgebras of powers, Transactions of the American Mathematical Society 362(3) (2010), 1445-1473.

DOI: https://doi.org/10.1090/S0002-9947-09-04874-0

[2] A. A. Bulatov and V. Dalmau, Towards a dichotomy theorem for the counting constraint satisfaction problem, Information and Computation 205(5) (2007), 651-678. 
[3] A. Chicco, On the primeness of locally finite idempotent 3-permutability, Algebra Universalis 80(2) (2019); Article 18.

DOI: https://doi.org/10.1007/s00012-019-0594-4

[4] A. Chicco, Prime Maltsev Conditions and Congruence $n$-Permutability, PhD. Thesis, McMaster University, Ontario, Canada, 2018.

[5] O. C. Garcia and W. Taylor, The lattice of interpretability types of varieties, Memoirs of the American Mathematical Society, American Mathematical Society Series 50(305) (1984).

DOI: http://dx.doi.org/10.1090/memo/0305

[6] J. Hagemann and A. Mitschke, On n-permutable congruences, Algebra Universalis 3(1) (1973); Article 8.

DOI: https://doi.org/10.1007/BF02945100

[7] D. Hobby and R. McKenzie, The Structure of Finite Algebras, Contemporary Mathematics, American Mathematical Society, 1988.

[8] K. A. Kearnes and A. Szendrei, Cube term blockers without finiteness, Algebra Universalis 78(4) (2017), 437-459.

DOI: https://doi.org/10.1007/s00012-017-0476-6

[9] K. A. Kearnes and S. T. Tschantz, Automorphism groups of squares and of free algebras, International Journal of Algebra and Computation 17(3) (2007), 461-505.

DOI: https://doi.org/10.1142/S0218196707003615

[10] K. A. Kearnes, Congruence permutable and congruence 3-permutable locally finite varieties, Journal of Algebra 156(1) (1993), 36-49.

DOI: https://doi.org/10.1006/jabr.1993.1061

[11] M. Kozik, A. Krokhin, M. Valeriote and R. Willard, Characterizations of several Maltsev conditions, Algebra Universalis 73(3-4) (2015), 205-224.

DOI: https://doi.org/10.1007/s00012-015-0327-2

[12] A. I. Maltsev, On the general theory of algebraic systems, Matematicheskii Sbornik 77(1) (1954), 3-20.

[13] R. McKenzie, G. McNulty and W. Taylor, Algebras, Lattices, Varieties, Volume 1, Wadsworth and Brooks, Cole, 1987.

[14] W. D. Neumann, On Malcev conditions, Journal of the Australian Mathematical Society 17(3) (1974), 376-384.

DOI: DOI: https://doi.org/10.1017/S1446788700017122

[15] J. Oprsal, Taylor's modularity conjecture and related problems for idempotent varieties, Order 35(3) (2018), 433-460.

DOI: https://doi.org/10.1007/s11083-017-9441-4 
[16] E. T. Schmidt, Kongruenzrelationen Algebraischer Strukturen, Mathematische Forschungsberichte 25 (1969).

[17] J. W. Snow, Maltsev conditions and relations on algebras, Algebra Universalis 42(4) (1999), 299-309.

DOI: https://doi.org/10.1007/s000120050005

[18] W. Taylor, Characterizing Mal'cev conditions, Algebra Universalis 3(1) (1973); Article 351.

DOI: https://doi.org/10.1007/BF02945141

[19] W. Taylor, The fine spectrum of a variety, Algebra Universalis 5(1) (1975), 263-303.

DOI: https://doi.org/10.1007/BF02485261

[20] S. Tschantz, Congruence Permutability is Join Prime, Unpublished, pages 1-70, 1996.

[21] M. Valeriote and R. Willard, Idempotent $n$-permutable varieties, Bulletin of the London Mathematical Society 46(4) (2014), 870-880.

DOI: https://doi.org/10.1112/blms/bdu044 